PNNL-11415

UC-713

\title{
A Portable Neutron Coincidence Counter
}
A. J. Peurrung
S. M. Bowyer
R. A. Craig
G. B. Dudder
M. A. Knopf

November 1996

Prepared for

the U.S. Department of Energy

under Contract DE-AC06-76RLO 1830

\author{
M. E. Panisko \\ P. L. Reeder \\ D. C. Stromswold \\ D. S. Sunberg
}




\section{DISCLAMMER}

Portions of this document may be illegible in electronic image products. Images are produced from the best available original document. 


\section{DISCLAIMER}

This report was prepared as an account of work sponsored by an agency of the United States Government. Neither the United States Government nor any agency thereof, nor any of their employees, make any warranty, express or implied, or assumes any legal liability or responsibility for the accuracy, completeness, or usefulness of any information, apparatus, product, or process disclosed, or represents that its use would not infringe privately owned rights. Reference herein to any specific commercial product, process, or service by trade name, trademark, manufacturer, or otherwise does not necessarily constitute or imply its endorsement, recommendation, or favoring by the United States Government or any agency thereof. The views and opinions of authors expressed herein do not necessarily state or reflect those of the United States Government or any agency thereof. 


\section{Summary}

Pacific Northwest National Laboratory has designed and constructed a prototype portable neutron coincidence counter intended for use in a variety of applications, such as the verification and inspection of weapons components, safety measurements for novel and challenging situations, portable portal deployment to prevent the transportation of fissile materials, uranium enrichment measurements in hard-to-reach locations, waste assays for objects that cannot be measured by existing measurement systems, and decontamination and decommissioning. The counting system weighs less than $40 \mathrm{~kg}$ and is composed of parts each weighing no more than $5 \mathrm{~kg}$. In addition, the counter's design is sufficiently flexible to allow rapid, reliable assembly around containers of nearly arbitrary size and shape. The counter is able to discern the presence of $1 \mathrm{~kg}$ of weapons-grade plutonium within an ALR-8 (30-gal drum) in roughly 100 seconds and $10 \mathrm{~g}$ in roughly 1000 seconds. The counter's electronics are also designed for maximum adaptability, allowing operation under a wide variety of circumstances, including exposure to gamma-ray fields of $1 \mathrm{R} / \mathrm{h}$. This report provides a detailed review of the design and construction process. Finally, preliminary experimental measurements that confirm the performance capabilities of this counter are discussed. 



\section{Contents}

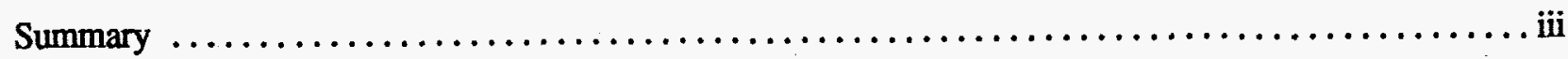

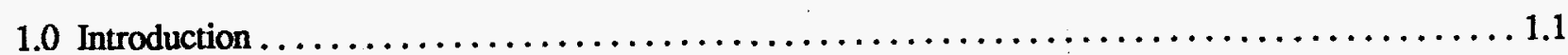

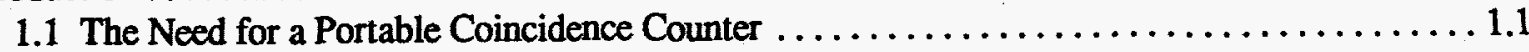

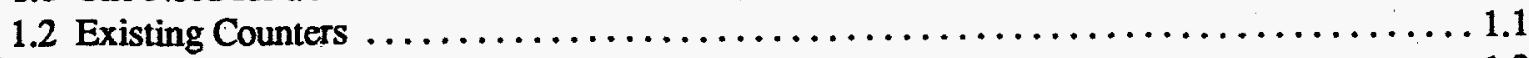

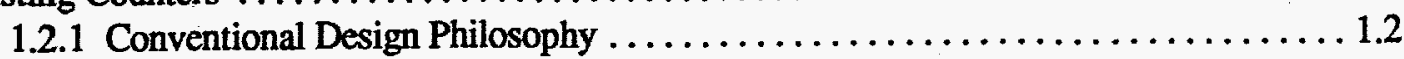

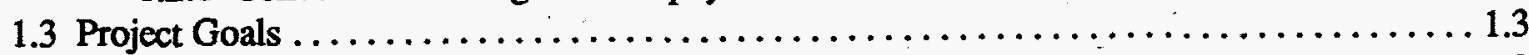

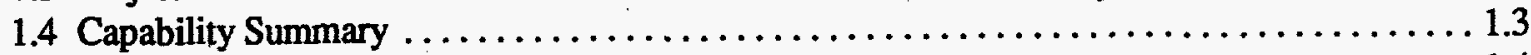

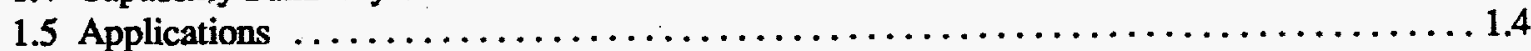

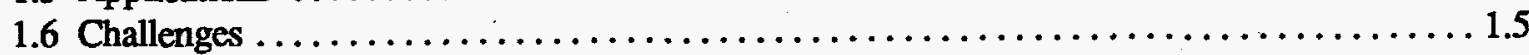

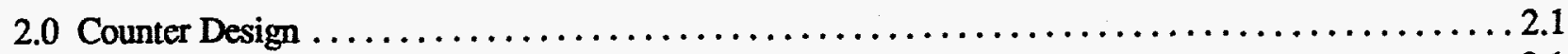

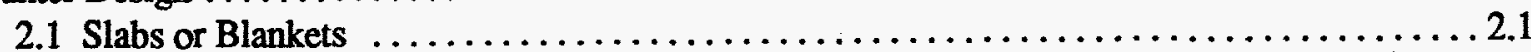

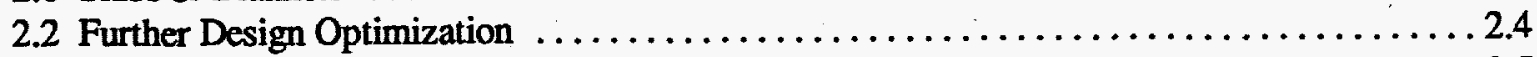

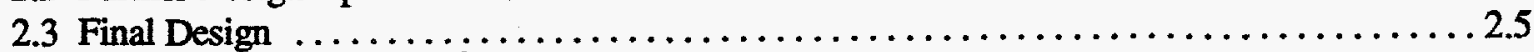

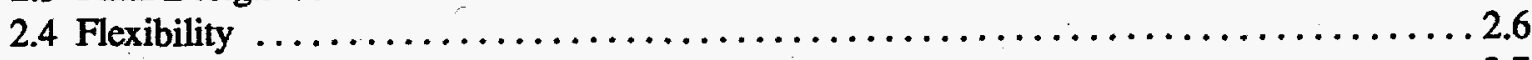

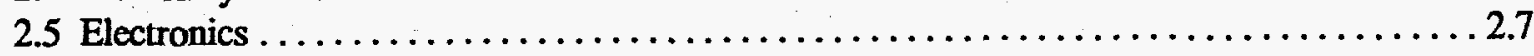

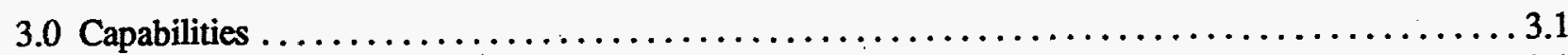

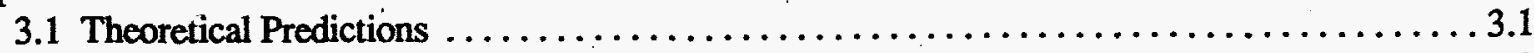

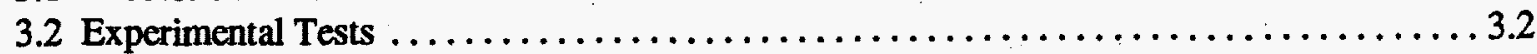

3.2.1 Effect of Multiple Wedges on Detection Efficiency $\ldots \ldots \ldots \ldots \ldots \ldots \ldots \ldots .2$

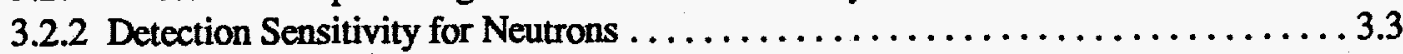

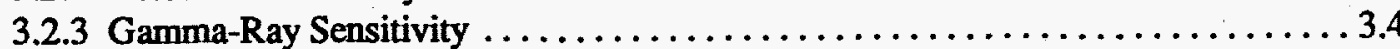

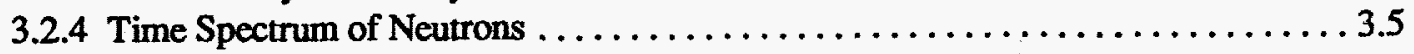

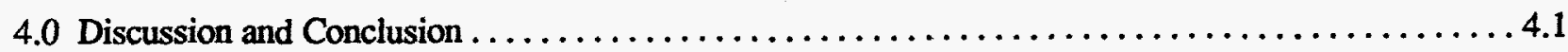

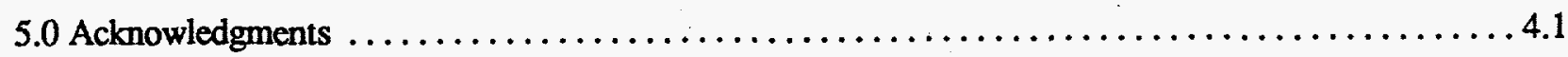

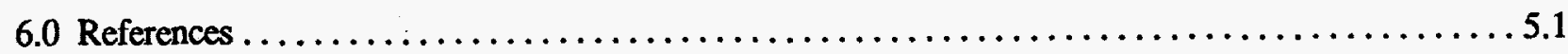

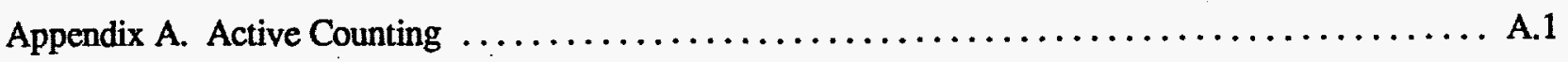

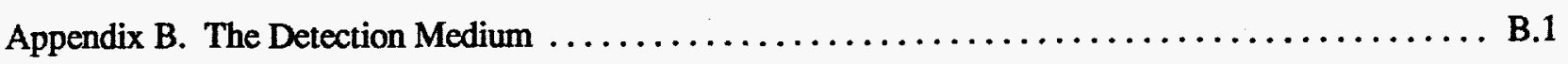

Appendix C. Optimization of Fiber-Detector Design $\ldots \ldots \ldots \ldots \ldots \ldots \ldots \ldots \ldots \ldots \ldots \ldots \ldots \ldots \ldots$ 


\section{Figures}

Figure 2.1 Simplified Sketch of Two Possible Ways to Use a Fixed Amount of Moderator in Constructing a

Portable Coincidence Counter ....................................

Figure 2.2. Normalized Histogram of Capture Probability as a Function of the Time Interval Between

Neutron Emission and Capture for Two Different Moderator Arrangements $\ldots \ldots \ldots \ldots \ldots \ldots 2.2$

Figure 2.3. Theoretical Efficiency of Traditional "Moderate-and-Capture" Neutron Detectors as a Function

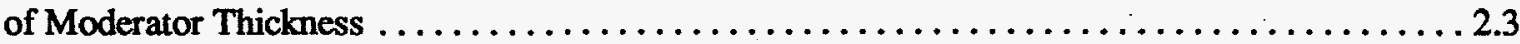

Figure 2.4 Probability of Capture in a Detector System with Moderator That Is "Folded" Three Times . . 2.3

Figure 2.5 Efficiency of 2.5-cm-Thick and 1.25-cm-Thick Detecting Slabs as a Function of the Quantity of

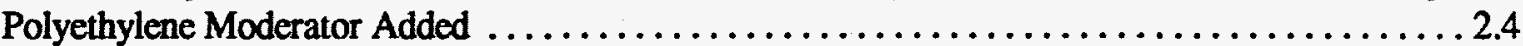

Figure 2.6 Efficiency of a 10-cm-Thick Moderating Detector as a Function of the Center-to-Center Spacing

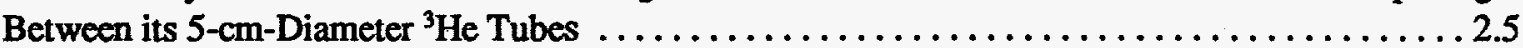

Figure 2.7 Stylized Diagram of the Final Detector Design $\ldots \ldots \ldots \ldots \ldots \ldots \ldots \ldots \ldots \ldots \ldots \ldots \ldots$

Figure 2.8. Typical Response of ${ }^{3}{ }^{3} \mathrm{He}$ Tube in a High Gamma-Ray Background $\ldots \ldots \ldots \ldots \ldots \ldots \ldots$

Figure 2.9 Neutron Pulse Shapes for a Variety of Pulse Shaping Times $\ldots \ldots \ldots \ldots \ldots \ldots \ldots \ldots . . \ldots$

Figure 2.10 Histogram of All Time Intervals after Each Pulse in $\mathrm{a}^{252} \mathrm{Cf}$ Neutron Pulse Train . . . . . 2.10

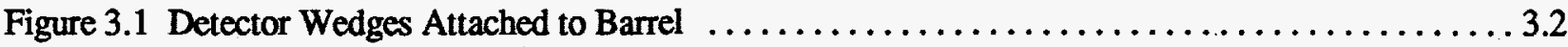

Figure 3.2 Electronics for Neutron Detection Efficiency Tests with Shift Register . . . . . . . . . . 3.3

Figure 3.3 Gamma-Ray Count Rate of ${ }^{3} \mathrm{He}$ Detector for Exposure to Gamma Rays . . . . . . . . . . 3.5

Figure 3.4 Time Spectrum from AmBe Showing Neutrons that Are Uncorrelated in Time ........... 3.6

Figure B.1 False Neutron Count Rate in a Set of ${ }^{10} \mathrm{BF}_{3}$ Tubes as a Function of Gamma-Ray Exposure

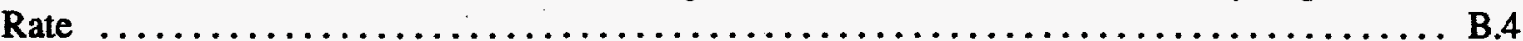

Figure C.1 Normalized Fiber Detector Efficiency as a Function of the Detector's Normalized ${ }^{6} \mathrm{Li}$ Content for

Four Different Thicknesses . ...............................

Figure C.2 Ratio of ${ }^{6} \mathrm{Li}$ Capture Probability to Hydrogen Capture Probability as a Function of the

Normalized ${ }^{6} \mathrm{Li}$ Detector Content for a 2.5-cm-Thick Detector ................. C.2

Figure C. ${ }^{6} \mathrm{Li}$ and Hydrogen Capture Probability as a Function of Optical Fiber Arrangement. The

efficiency of a detector is essentially the same as the probability for ${ }^{6} \mathrm{Li}$ capture $\ldots \ldots \ldots \ldots \ldots$ C.3

\section{Tables}

Table 3.1 Effect of Multiple Detector Wedges on Count Rate $\ldots \ldots \ldots \ldots \ldots \ldots \ldots \ldots \ldots \ldots \ldots \ldots$

Table 3.2 Neutron Counts Obtained from Detector Wedges Using Shift Register . . . . . . . . . . . . 3.4

Table B.1 Gamma-Ray Response Data for Five Different Fiber-Based Neutron Counters ......... B.5 


\subsection{Introduction}

\subsection{The Need for a Portable Coincidence Counter}

Neutron coincidence counting is an established method for controlling and confirming the presence of a wide range of fissile materials. Simply put, any material that undergoes either spontaneous or induced fission must be a special nuclear material. The only conceivable way to mimic the presence of a particular fissile material would be with some quantity of alternate fissile materials. In addition, it is difficult to suppress neutron radiation using shielding materials. An effective neutron shield would necessarily have a weight and size such that covert material movement would be hard to disguise.

Coincident neutron counting involves recording the number of neutron pairs likely to have originated from the same fission event. A coincidence counter is, of course, generally capable of performing simultaneous singles neutron counting to yield additional information about the unknown materials. Coincidence counting has several advantages for assaying fissile materials:

- The emission of multiple neutrons during a single event constitutes a strong signature for nuclear fission. The only "natural" event which mimics nuclear fission is cosmic ray spallation, in which a nucleus is fragmented upon interaction with an energetic cosmic ray. The cosmic ray spallation rate is generally known and constitutes a small and steady background that can be subtracted.

- Since the signal depends on the detection efficiency squared, coincidence counting provides relatively high background rejection, i.e., rejection of those fission events that do not occur within the intended container.

- Coincidence counters measure both the desired signal and the dominant accidental background simultaneously. Thus, it is not necessary to perform a separate background count for subtraction. This not only saves time and increases accuracy, but also makes possible numerous measurements for which a "clean" background count would not be possible. For example, suppose that it is necessary to assay a large, immovable container for fissile materials. Conventional singles counting requires the counting of a "clean" container with similar size, mass, and composition at the location of the "dirty" container with the "dirty" container well removed. This is clearly impossible for many applications.

A considerable need exists for a coincidence counter that is portable, modular, flexible, and adaptable to support safeguards, transparency, and irreversibility initiatives. Such a device would greatly lower the cost associated with any awkward, remote, or unique nondestructive assay requirements by providing sufficient accuracy for inherently low-intrusion inspections. The counter described in this report eliminates these drawbacks by sacrificing efficiency to allow the construction of an instrument that is lightweight and can be rapidly assembled around any existing container or structure.

\subsection{Existing Counters}

Excellent instruments exist currently that are capable of highly effective coincidence counting. (Fehlau et al. 1990; Armitage et al. 1994) (Canberra Industries, Inc., Nuclear Products Group, 800 Research 
Parkway, Meriden, Connecticut 06450). However, the design philosophy for these instruments differs greatly from that of the instrument described in this report.

\subsubsection{Conventional Design Philosophy}

Because many applications require the utmost in accuracy and speed, neutron detection efficiency is often the primary goal for counter design. These counters are capable of achieving $30 \%$ or more detection efficiency for singles neutron counting. The following is an itemized list of the general advantages and disadvantages of such designs:

\section{Advantages}

- Precision: Increased efficiency leads to high count rates, allowing data to be acquired with excellent statistics. The smallest possible experimental uncertainties are often required for applications such as transuranic (TRU) waste assay or fissile material assay.

- Speed: High efficiency and good statistics will also make rapid assay feasible. Some waste assay applications or safeguards applications involve a very large number of assay measurements. A counter for these applications must be fast to be economically competitive.

- Accuracy: The high efficiency of many neutron counters leads to the acquisition of data with sufficient quality to allow extraction of additional information, such as matrix composition, chemical form, isotopic makeup, or internal multiplication. This additional information can lead to estimated fissile masses that are significantly less susceptible to systematic errors (Dytlewski et al. 1993).

\section{Disadvantages}

- Portability: Also for reasons of weight, transporting conventional counting systems is difficult and sometimes impossible.

- Flexibility: Such neutron counters are generally too intricate, thick, and heavy to permit a flexible design. Consequently, many systems are intended for use with only a single type of container (or with containers of a small size.)

- Adaptability: The same elaborate apparatus and methods that allow high-precision assays often preclude the use of a particular counter for new and substantially different measurement problems. Only a modular counter is appropriate for measurement problems where a particularly high or low efficiency is needed.

- Cost: High efficiency counters tend to require the use of a large quantity of neutron detectors (such as ${ }^{3} \mathrm{He}$ tubes), which leads to a generally higher cost. 


\subsection{Project Goals}

Listed below are the four main goals for our project to construct a portable neutron coincidence counter:

- Portability: Our primary design criterion was portability. More specifically, we wanted the final counting system to weigh less than $40 \mathrm{~kg}$ and to consist of pieces weighing less than $5 \mathrm{~kg}$ each. These specifications should lead to a counting system that travels to the container, rather than a counter that requires the container to be brought to it.

- Flexibility: Along with portability, we have designed a counting system with maximum physical flexibility. The counter is modular and capable of assembly around objects with widely varying shapes and sizes. The assayed objects need not be "containers" at all, but rather may be process piping or other hardware. Because a physically flexible counting system is likely to be put to a relatively wide variety of uses, electronic and software flexibility will also be required.

- Gamma-Ray Response: This counter must be able to perform nonintrusive weapons component verification and inspection. These applications require a significant exposure to gamma-ray fields. Thus, we have decided that the counter should be capable of operation during exposure to gammarays at rates up to $1 \mathrm{R} / \mathrm{h}$.

- Active Counting: A portable neutron coincidence counter capable of active counting would fill a critical need in the user community. (Active counting uses a separate neutron source for uranium assay via measurement of the induced fission rates.) Appendix A discusses our conclusion that constructing a portable active coincidence counter with current technology would be exceedingly difficult. The counting system discussed in this report operates only in the passive mode.

The design goals listed above represent a significant change in design philosophy compared to conventional coincidence counters. Portability, rather than accuracy, precision, and speed, is regarded as the primary design criterion. Efficiency must, of course, be maximized, but only subject to the portability and flexibility constraints outlined above. In addition, we have endeavored to demonstrate that a counter constructed with these design criteria will have sufficient accuracy and precision to retain significant utility for a variety of applications.

\subsection{Capability Summary}

The specific measurement capabilities of the counting system that has been designed and built are detailed in Section IV. For any particular container, it is desired that the singles neutron-counting efficiency is at least $1 \%$ so that the efficiency for counting coincident pairs of neutrons will be at least $10^{-4}$. Because achieving this performance will require an amount of moderator (and quantity of neutron detectors) that depend on the container size and shape, the detector design is modular. The specified overall system weight of $40 \mathrm{~kg}$ is assumed to apply to a counting system that is assembled around an ALR-8 container (30-gal drum). Theoretical calculations predict that a system with these counting efficiencies should be able to confirm the presence of 10 grams of weapons-grade plutonium within an ALR-8 in 1000 seconds ( ${ }^{40} \mathrm{Pu}$ is the primary source of neutrons in weapons-grade plutonium). 
The prototype counting system constructed has been experimentally shown to achieve $>1.5 \%$ efficiency for detecting ${ }^{252} \mathrm{Cf}$ spontaneous fission neutrons within a 30 -gal drum. The weight of the detection components necessary to achieve this efficiency is $33 \mathrm{~kg}$, although this does not currently include the weight of any coincidence counting electronics (final versions of these electronics should not add too much to the weight, but are not yet implemented).

\subsection{Applications}

This counter should have a general use whenever remote, awkward, or unique measurements are required. It is our hope that completion of this counter's development will result in a significant reduction in the long-term research and development costs associated with new neutron-counting systems. Specific application areas are listed below:

- Weapons component verification and inspection: A portable coincidence counter may be ideally suited for non-intrusive inspections of weapons components. The goal of the assay would be to establish the presence and general nature of the weapons components inside a sealed container. Inspections may often be required in remote locations. Moving the container will often be undesirable for reasons of health and safety.

- Safeguards: Even though the existing series of neutron counters performs satisfactory safeguards measurements every day in a variety of facilities, a number of material assay problems remain unsolved owing to the difficulty of using "single purpose" counters for novel and challenging tasks. Measurements performed with a portable coincidence counter should have significant value despite lower accuracy and precision simply because no other counter is capable of doing the job.

- Portable portal deployment: During crises, it may be necessary to rapidly assemble and operate a "portal" designed to prevent the passage of fissile material. A flexibly-designed, portable neutron coincidence counter may be well suited to this task.

- Uranium enrichment: Problems in uranium enrichment measurement sometimes require a neutron counting system capable of being transported to hard-to-reach areas and then wrapped around process piping. The portable, flexible system developed during this project would be ideally suited to such applications (Stromswold et al. 1996).

- Waste assay: Although this system is not capable of the highly accurate and precise measurements that heavier systems offer, some waste objects are of a size and shape that cannot be assayed at all using existing systems. A portable, flexible, and modular neutron counting system may offer an ideal solution to such problems. The assays would not be rapid, but would not need to be since the inventory of such oddly shaped and sized equipment is not as substantial as the inventory of standard barrels and boxes.

- Decontamination/decommissioning (D\&D): Measuring plutonium holdup in process piping is an important step in any D\&D effort. Because it can be assembled around oddly shaped objects, the portable neutron counter described below should find applications in these areas. In addition, the measurement of holdup is essential for materials accounting purposes. 


\subsection{Challenges}

Calibrating a portable neutron coincidence counter will be the most important technical obstacle in completing its development. Possible sources of systematic error must be understood and, if possible, controlled before the assay results from such a counter will have sufficient reliability. Several sources of systematic error are listed below:

- Matrix effects: The matrix (non-fissile material) inside a container affects the outgoing neutron energy spectrum, which in turn affects the counter efficiency and assay result. It will be necessary to optimize the counter design to minimize such effects and/or find a method to explicitly compensate for matrix effects.

- Geometry effects: Because the shape of the counter itself changes from measurement to measurement, the counter's efficiency will vary. It will be necessary to develop methods to minimize the uncertainty that this adds to the ultimate assay results.

- Locational effects: The precise location of the fissile material within a container may not be known. Changes in material location may alter the counter efficiency and, therefore, assay results. Data analysis techniques capable of compensating for these effects must be developed and demonstrated.

- Self-multiplication effects: Large quantities of fissile material may lead to neutron multiplication, which alters the relationship between coincident count rate and fissile material mass. Methods must be developed to compensate for these effects, or at least identify those cases for which multiplication may be important. 


\subsection{Counter Design}

\subsection{Slabs or Blankets}

One issue in designing a portable coincidence counter is how to achieve the desired neutron detection efficiency. The imposed weight limit fixes the total amount of neutron moderator that can be used in the device. Compared to the moderator, all other system components are relatively light. Exceptions to this statement occur when an environmental moderator can be used, but this is not the case for this coincidence counting application. Figure 2.1 shows two different moderator arrangements that might be considered for constructing a coincidence counter. Should the moderator consist of discrete slabs of moderator that are relatively thick, or should it consist of a single "blanket" that is uniformly thin? It is critical to understand which of these forms is the appropriate detection technology for constructing a portable coincidence counter.
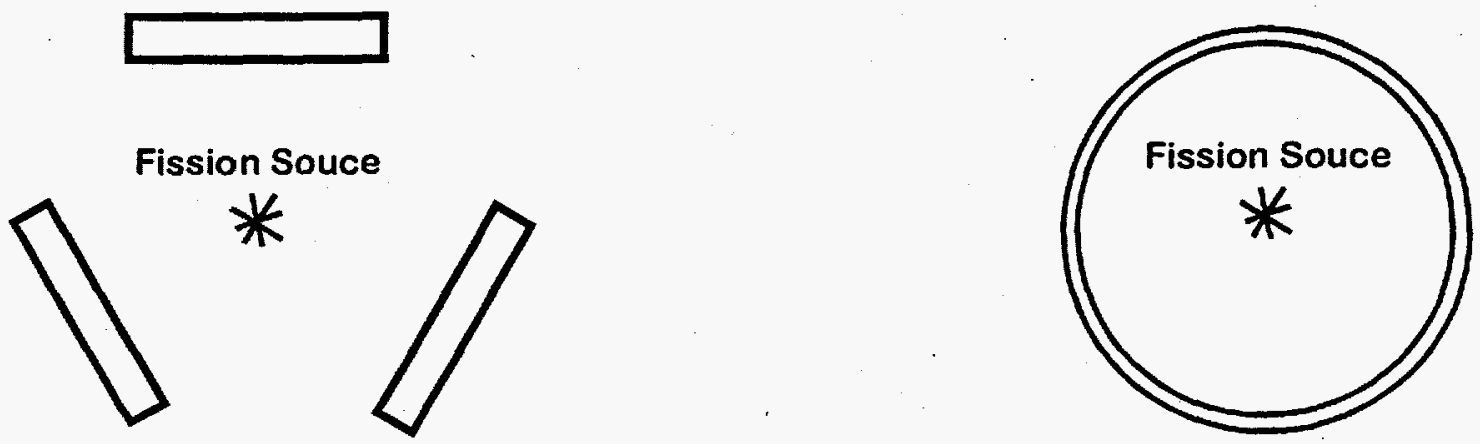

Figure 2.1 Simplified Sketch of Two Possible Ways to Use a Fixed Amount of Moderator in Constructing a Portable Coincidence Counter

Numerical modeling of neutron transport using the well-benchmarked Monte Carlo N-particle transport code (MCNP) (Briesmeister 1993) reveals two reasons why a fixed amount of moderator is best used as discrete slabs rather than as a "blanket." The slab geometry has both superior neutron detection efficiency and timing characteristics.

Figure 2.2 shows a histogram of the time between emission and capture for a centrally located point source of fission neutrons that is surrounded by two different moderator arrangements. Both curves are normalized by the neutron detection efficiency for comparison (see the subsequent bullet for efficiency information.) This figure clearly shows that the time intervals between neutron emission at the source and neutron capture in the detector are both longer and more variable for a thin-sheet moderator. A neutron counting system would require a significantly larger coincidence timing window to detect such neutrons efficiently. This larger timing window, in turn, would result in a higher accidental coincidence background and correspondingly poorer performance. Timing considerations, therefore, argue for the use of a moderator in relatively thick segments that cover less solid angle with respect to the neutron source.

As shown in Figure 2.3, the detection efficiency achieved by a planar section of polyethylene depends in a highly nonlinear way on thickness. This is physically reasonable since the process of neutron moderation and capture is essentially a diffusion process. (A neutron typically undergoes 25 to 30 separate collisions within the moderator before undergoing capture.) We conclude that "folding" a thin sheet of moderator upon 
itself so that its area is halved but its thickness doubled results in a significant increase in efficiency. Figure 2.4 demonstrates the numerically calculated advantage of "folding" a thin $1.25 \mathrm{~cm}$ thick spherical moderator three times so that it becomes first a hemisphere, then a quadrant (solid angle $=\pi$ ), and finally an octant (solid angle $=\pi / 2$.) Note that three "foldings" results in an efficiency gain of greater than a factor of 10 without any change in the total detector weight. Again, this calculation assumes that a fixed quantity of neutron capture agent such as ${ }^{3} \mathrm{He}$ is uniformly distributed within the moderator.

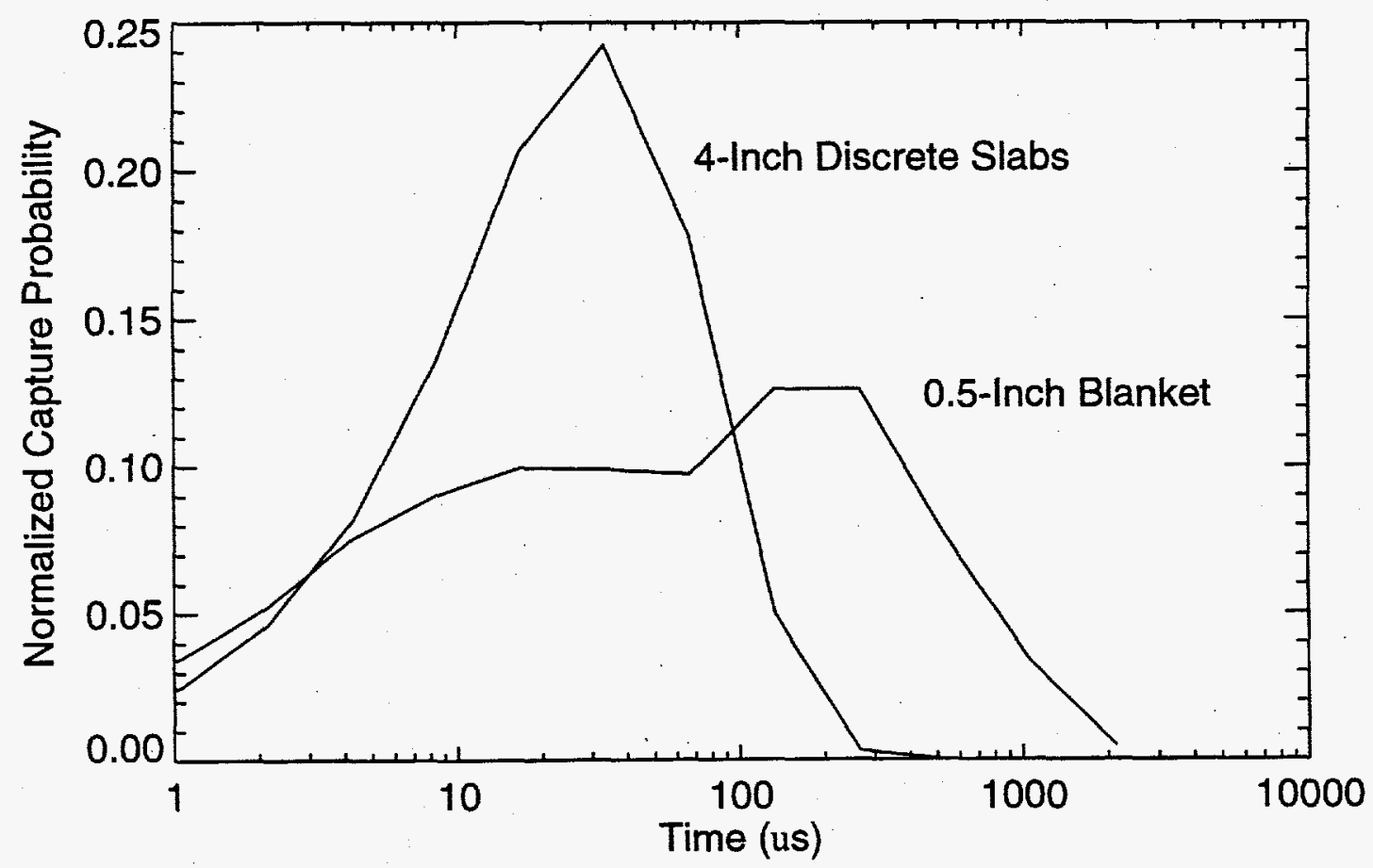

Figure 2.2. Normalized Histogram of Capture Probability as a Function of the Time Interval Between Neutron Emission and Capture for Two Different Moderator Arrangements. The taller curve applies to a 10-cm-thick system covering $1 / 8$ of the solid angle with respect to the neutron source. The lower curve applies to a1.25-cm-thick system that completely covers the solid angle. 


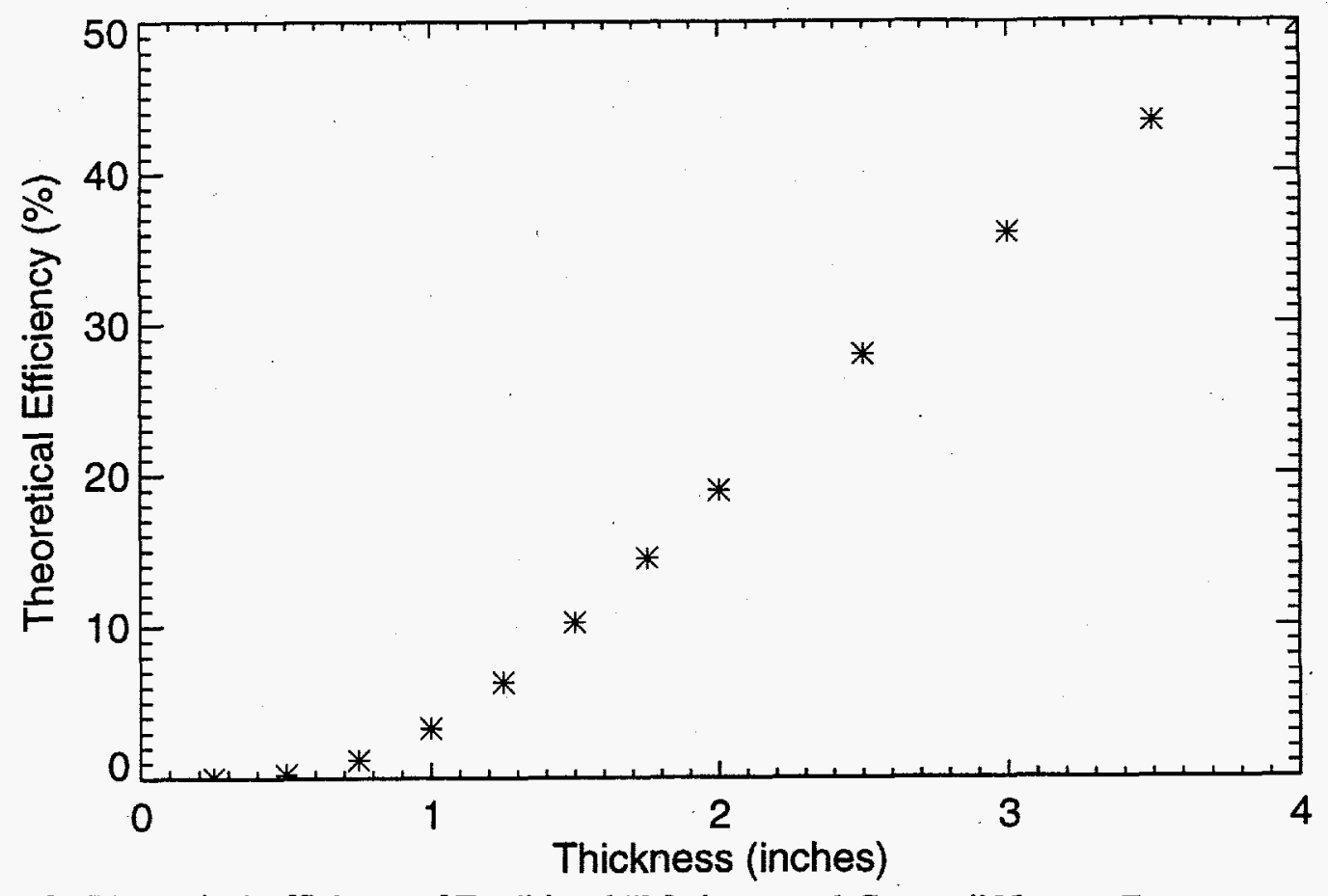

Figure 2.3. Theoretical Efficiency of Traditional "Moderate-and-Capture" Neutron Detectors as a Function of Moderator Thickness. The calculations used to generate the data in this figure assume that a neutron capture agent such as ${ }^{6} \mathrm{Li}$ or ${ }^{3} \mathrm{He}$ is distributed evenly throughout an infinite-slab-shaped detector (moderator). Neutrons are assumed to strike the slab at normal incidence with a fission spectrum of energies.

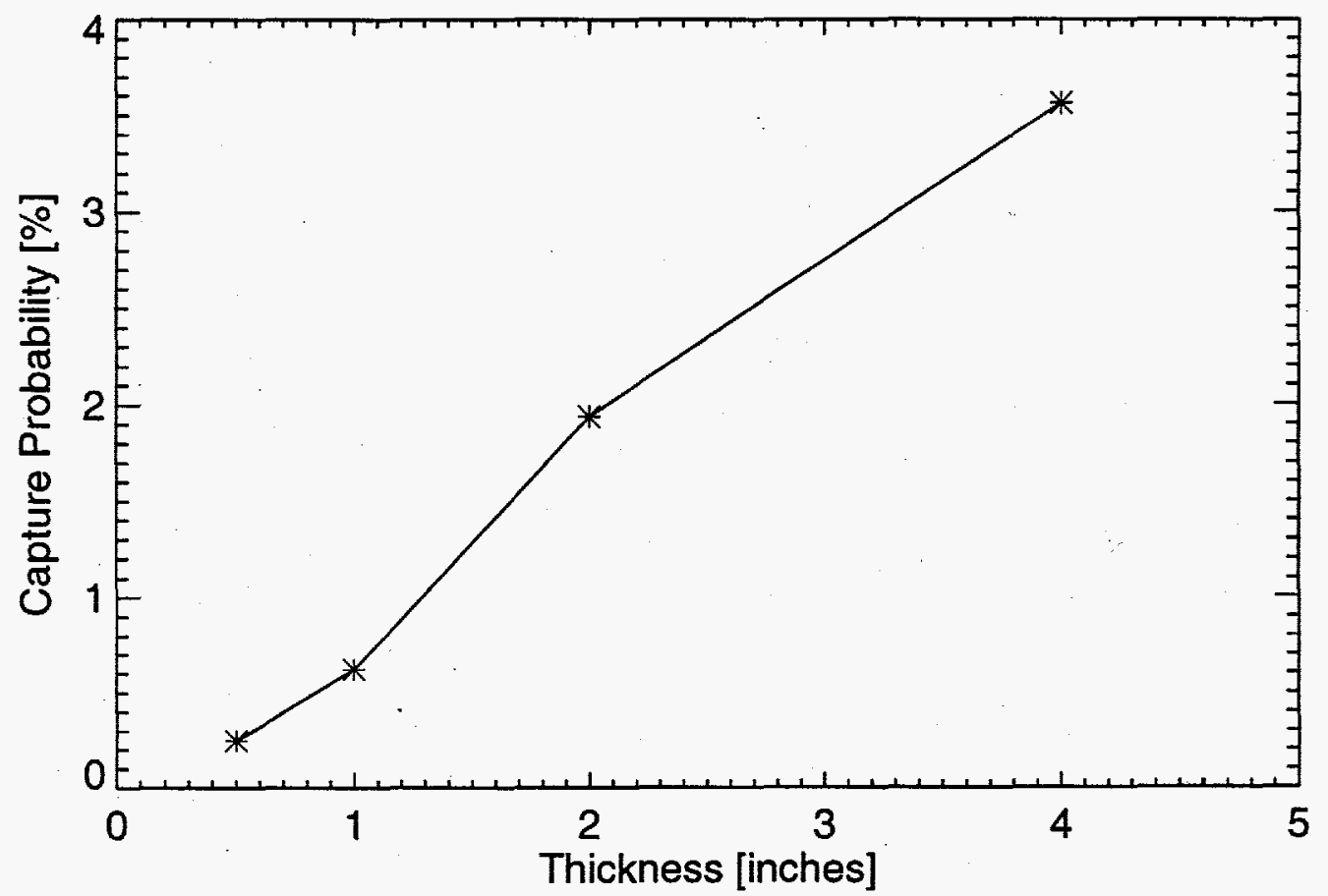

Figure 2.4 Probability of Capture in a Detector System with Moderator That Is "Folded" Three Times. Each time that the moderator is folded, the thickness doubles while the area is halved. The total amount of moderator (and ${ }^{3} \mathrm{He}$ ) is fixed. The capture probability is essentially a measure of the system's neutron detection efficiency. The $1.25-\mathrm{cm}$-thick moderator completely surrounds the neutron source. 


\subsection{Further Design Optimization}

The designs for both scintillating optical fiber and ${ }^{3} \mathrm{He}$ tube-based detectors were analyzed extensively. These computations were carried out using the well benchmarked MCNP neutron transport code, version 4A (Briesmeister 1993). This subsection describes several of the basic results of these computations. We did these computations so we could learn the principles of detector design well enough to design an adaptable counter with an excellent efficiency-to-weight ratio.

The results discussed in the previous subsection have already described two factors that have a dramatic impact on detector efficiency. First, the detector must not be very thin; 5 centimeters can be considered the minimum moderator thickness from which a useful detector can be constructed. Since a detector of this thickness that completely surrounds a large container would weigh far more than $50 \mathrm{~kg}$, the detector's design must incorporate discrete slabs, which can be placed at intervals around the container. A further reason to make use of discrete slabs is the discovery that a neutron will, on average, be moderated and captured more quickly with this arrangement. This is critical for any coincidence counter.

The efficiency of a relatively thin moderating detector can be dramatically improved by adding "backing" moderator. Figure 2.5 shows the efficiency of a planar detecting slab as a function of the quantity of polyethylene added. Backing moderator is simply attached to the outside of an existing detector segment. The backing moderator does not contain any neutron detectors. The added moderator may be simple and cheap; it may be stored at the site of the measurement and therefore not add to the detector's overall weight. Although our final detector design does not make use of such backing moderator, we wish to stress that it may be enormously useful for future counter design variations.

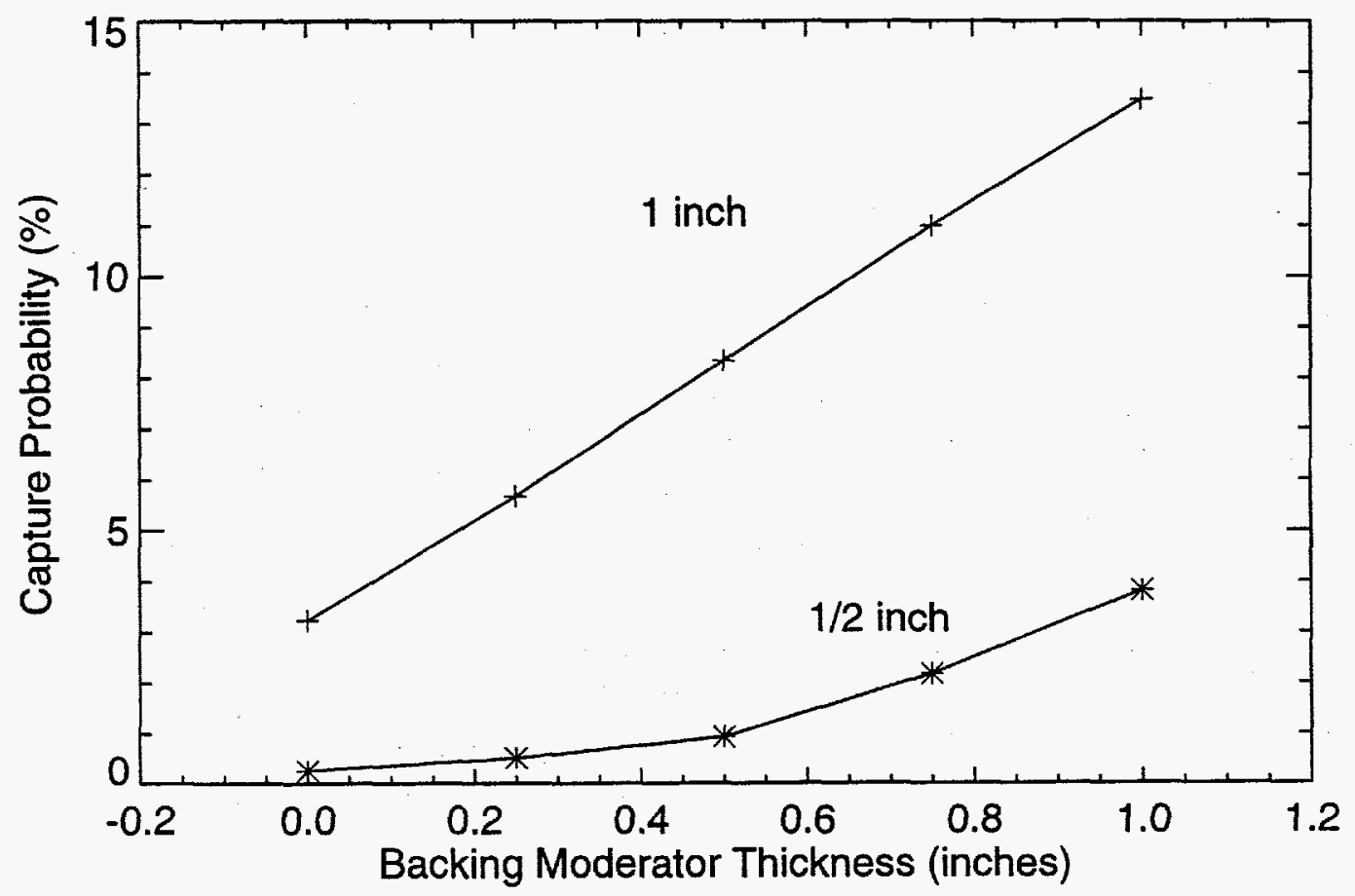

Figure 2.5 Efficiency of 2.5-cm-Thick and 1.25-cm-Thick Detecting Slabs as a Function of the Quantity of Polyethylene Moderator Added. For simplicity, neutrons with a uranium fission energy spectrum are assumed to strike a detector in the shape of an infinite plane. 
Appendix $\mathrm{C}$ describes the design optimization computations that were performed for neutron detecting systems constructed with ${ }^{6} \mathrm{Li}$-containing scintillating fibers rather than ${ }^{3} \mathrm{He}$ tubes. These calculations address the effect of varying the total ${ }^{6} \mathrm{Li}$ content and the arrangement of fibers within the moderating matrix.

Lastly, it was important to determine the efficiency of a moderating slab as a function of the spacing between adjacent ${ }^{3} \mathrm{He}$ tubes. Figure 2.6 shows the results of these calculations for a detector with a $10-\mathrm{cm}$ thickness. (The tubes are placed centrally within the moderator.) It is important to notice that efficiency is not especially sensitive to tube spacing. Similar results would be expected for other thicknesses.

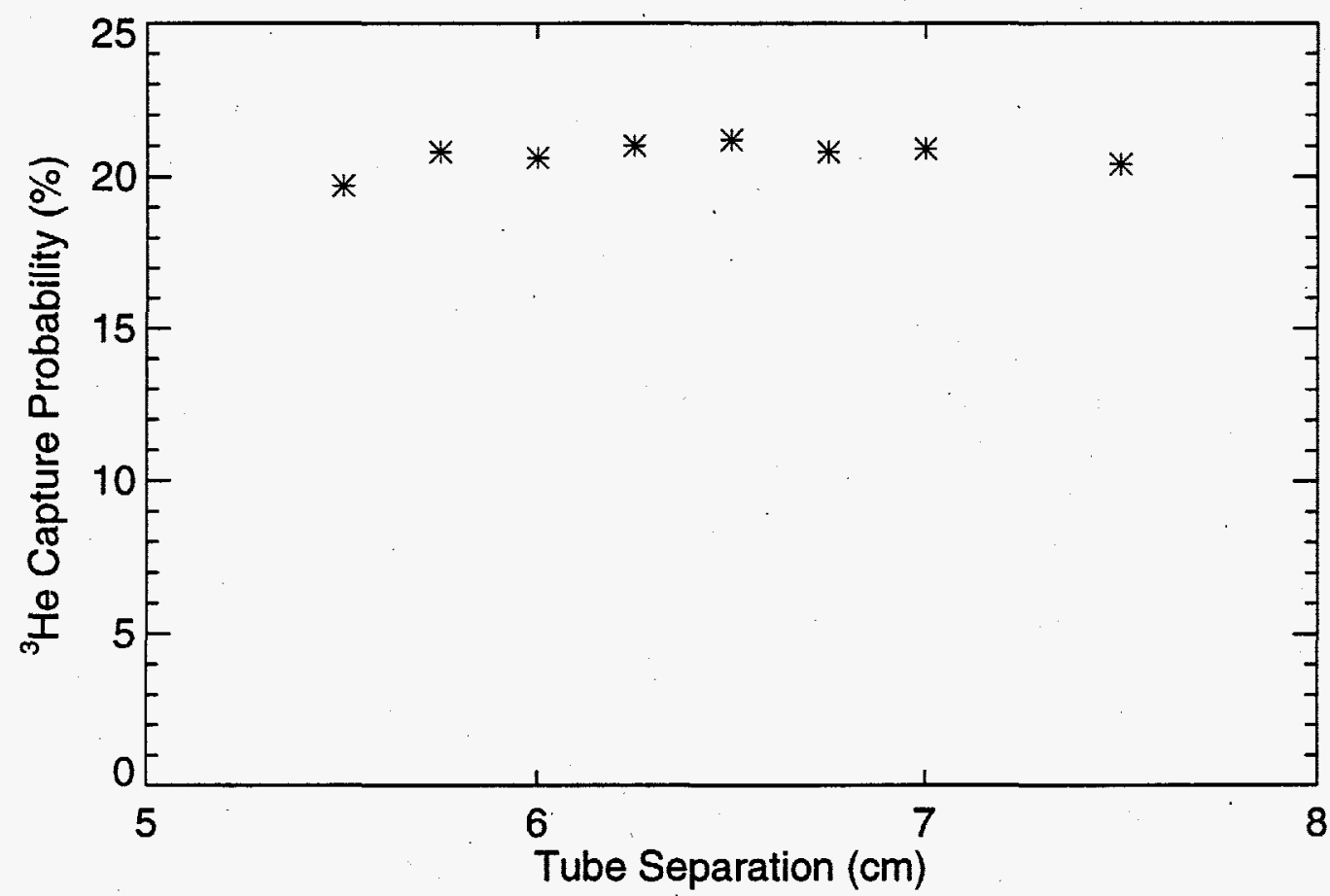

Figure 2.6 Efficiency of a 10-cm-Thick Moderating Detector as a Function of the Center-to-Center Spacing Between its 5-cm-Diameter ${ }^{3} \mathrm{He}$ Tubes. A uranium fission neutron source is assumed.

\subsection{Final Design}

A final design for the coincidence counter was selected in accordance with many of the results that have been discussed so far. Figure 2.7 contains a schematic diagram of the final detector design.

Rather than ${ }^{6} \mathrm{Li}$ scintillating fibers, ${ }^{3} \mathrm{He}$ tubes are used to allow operation in gamma-ray fields as high as $1 \mathrm{R} / \mathrm{h}$. A moderator that is $7.5 \mathrm{~cm}$ thick is used as a compromise that achieves reasonably high efficiency, but allows the weight of the individual pieces that make up the detector to be kept to around $5 \mathrm{~kg}$. The highest efficiency-to-weight ratio uses ${ }^{3} \mathrm{He}$ tubes with 5 -cm diameters. The detector has a design such that adjacent ${ }^{3} \mathrm{He}$ tubes are spaced by no less than $7.5 \mathrm{~cm}$ (center-to-center). Efficiency is increased by purchasing ${ }^{3} \mathrm{He}$ tubes filled with 4 atmospheres of ${ }^{3} \mathrm{He}$ rather than the more standard 2 atmospheres. Although this is added to the cost of the ${ }^{3} \mathrm{He}$ tubes, a relatively small number of tubes is required for constructing a detector of 
this sort. The active length of our ${ }^{3} \mathrm{He}$ tubes is approximately $85 \mathrm{~cm}$. There is enough moderator extending beyond the active region at both ends of the tube to make full use of the entire 85-cm-long active region. This length makes it possible to assay large containers and yet keeps the weight of individual detector segments to roughly $5 \mathrm{~kg}$. The numerically calculated efficiency of this detector design is approximately $12 \%$ (this is the efficiency for detecting neutrons that strike a planar moderating slab consisting of a large number of detector segments).

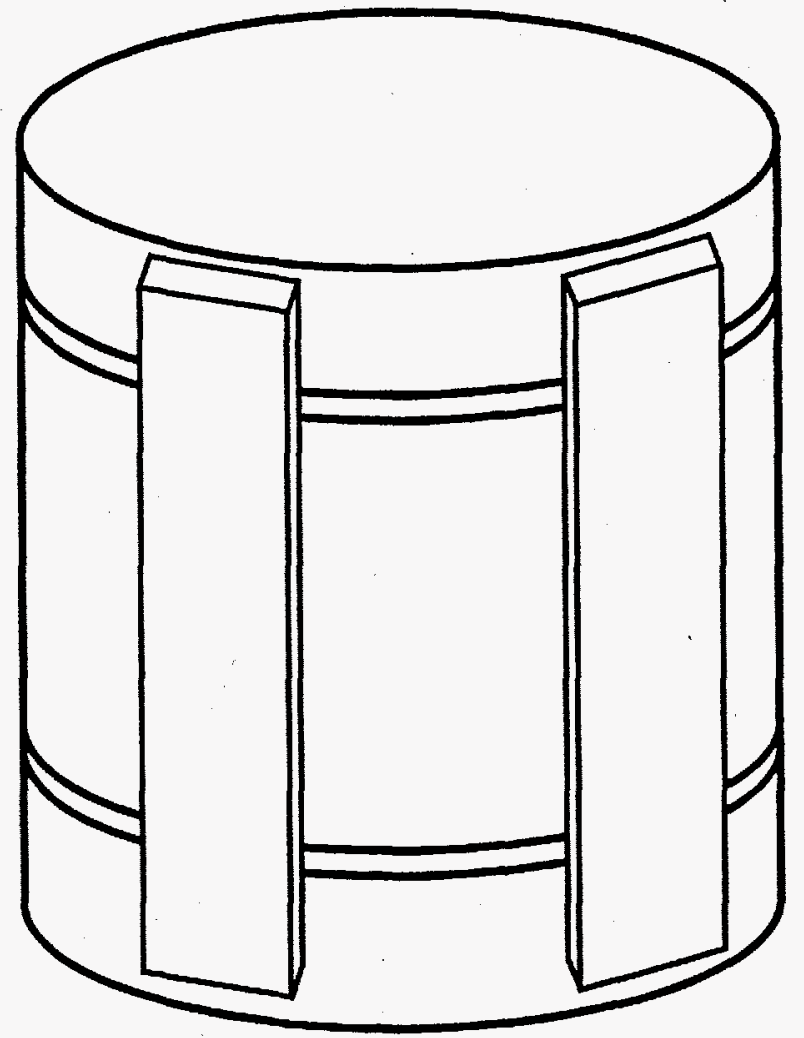

Figure 2.7 Stylized Diagram of the Final Detector Design

\subsection{Flexibility}

An important project goal was to design a detector with sufficient flexibility to assay containers of various shapes and sizes. Our design achieves this goal by allowing the end user to "attach" detector segments to a container as necessary. Each segment currently weighs $5.4 \mathrm{~kg}$ and contains a single ${ }^{3} \mathrm{He}$ tube. The sides of the segments are slanted somewhat to allow groups of segments to "bend" around a curved surface. Currently, our plan is that each segment will not attach to the container directly, but rather to a flexible, lightweight belt or cover that is fastened directly to the container. Although this detector assembly has no firm requirements, a number of recommendations are listed below:

- The segments should be used in groups whenever possible to increase efficiency. A single detector segment is not very efficient because neutrons readily escape from the sides of the segment without being detected. (Experiments indicate that 1,2 , and 3 segments placed in a group are $0.91 \%, 2.88 \%$, 
and $5.25 \%$ efficient, respectively, around an ALR-8 container.) Groups of 3 detector segments or more are ideal.

- The segments should be placed as close to the container as possible.

- The segments should be placed at three or four locations around the outside of a container.

- Enough segments should be placed around the container to achieve the desired efficiency and detector performance. It is anticipated that six segments will easily suffice for an ALR-8 (30-gal) drum.)

As discussed in the introduction, a number of important challenges remain before a detector assembled in this way will be trustworthy. Most neutron counters have a fixed shape and a fixed environment; such counters need only be calibrated and tested once. (Of course, operational checks will be needed to establish that the counter is functioning properly.) A flexible counter, however, requires a testing and calibration procedure that is as adaptable as the counter itself. Achieving reliable operation of this counter under a variety of conditions constitutes much of the remaining work.

\subsection{Electronics}

Two levels of electronics are necessary to operate the coincidence counter. First, signal-processing electronics are needed to amplify and shape neutron pulses. It is generally necessary that these electronics be located in close proximity to the detection devices. Additionally, it is best to completely discriminate between neutron and gamma-ray pulses independently at each ${ }^{3} \mathrm{He}$ tube before the signals are logically combined for coincidence analysis. For these reasons, a circuit has been developed that connects directly to the end of each ${ }^{3} \mathrm{He}$ tube and provides all of the necessary signal processing. The circuit is small and rugged and attaches firmly and seamlessly to the end of a 5-cm-diameter ${ }^{3} \mathrm{He}$ tube, adding several inches to its length.

Separating the signal processing functions for each tube is not sufficient to achieve a satisfactory response in gamma-ray fields as strong as $1 \mathrm{R} / \mathrm{h}$. This level of performance requires very fast pulse-shaping times. Figure 2.8 shows the mechanism by which a ${ }^{3} \mathrm{He}$ tube falsely records a neutron count in the presence of a high gamma-ray background. Clearly, the "accidental" pileup of small gamma-ray pulses can be reduced with the use of a shorter shaping time constant. Figure 2.9 shows typical neutron pulses for a variety of pulse-shaping time constants. We expect that using a time constant smaller than $1 \mu$ s will allow satisfactory tube operation in fields as strong as $1 \mathrm{R} / \mathrm{h}$.

The second level of electronics necessary to operate a coincidence counter is to analyze the incoming neutron pulse train for indications of a real coincident signal. Any method for analyzing this pulse train requires quantitative analysis of the time intervals between pulses. In essence, a mathematically verifiable correlation is sought between nearby pulses that would not be present in a train of neutron pulses from a single neutron source. If a histogram of the time intervals between each pulse and all of its following pulses is plotted, a graph similar to that shown in Figure 2.10 is obtained. These data were obtained using a ${ }^{252} \mathrm{Cf}$ source. Note that for large time intervals, the curve approaches the rate of accidental coincidences. The area under this curve (with the appropriate subtraction of "accidental" background) represents the coincident signal. The "shift register" method for analyzing coincidence data effectively performs this integration and background subtraction (Halbig et al. 1992). Because we have designed a counting system with the primary goals of flexibility and adaptability, a shift register with fixed integration bounds may not yield the optimum performance for every problem. Therefore, we have acquired a commercial time recording circuit that fits 
into a small personal computer (GT 655 Time Interval Analyzer). This circuit records the arrival time for all neutron pulses and periodically passes this information on to the computer's memory for software analysis. This system can be operated successfully, primarily because our relatively low-efficiency counter should not experience exceptionally high data rates. Another advantage of this system is that it permits an unprecedented degree of application flexibility. Should it be necessary to adapt the counting system to a completely new application, any changes to the data processing algorithm can be carried out with software. For example, this system could work with a neutron generator or other modulated neutron source to reduce the background. Additionally, it could be quickly reprogrammed to function as a multiplicity counter.

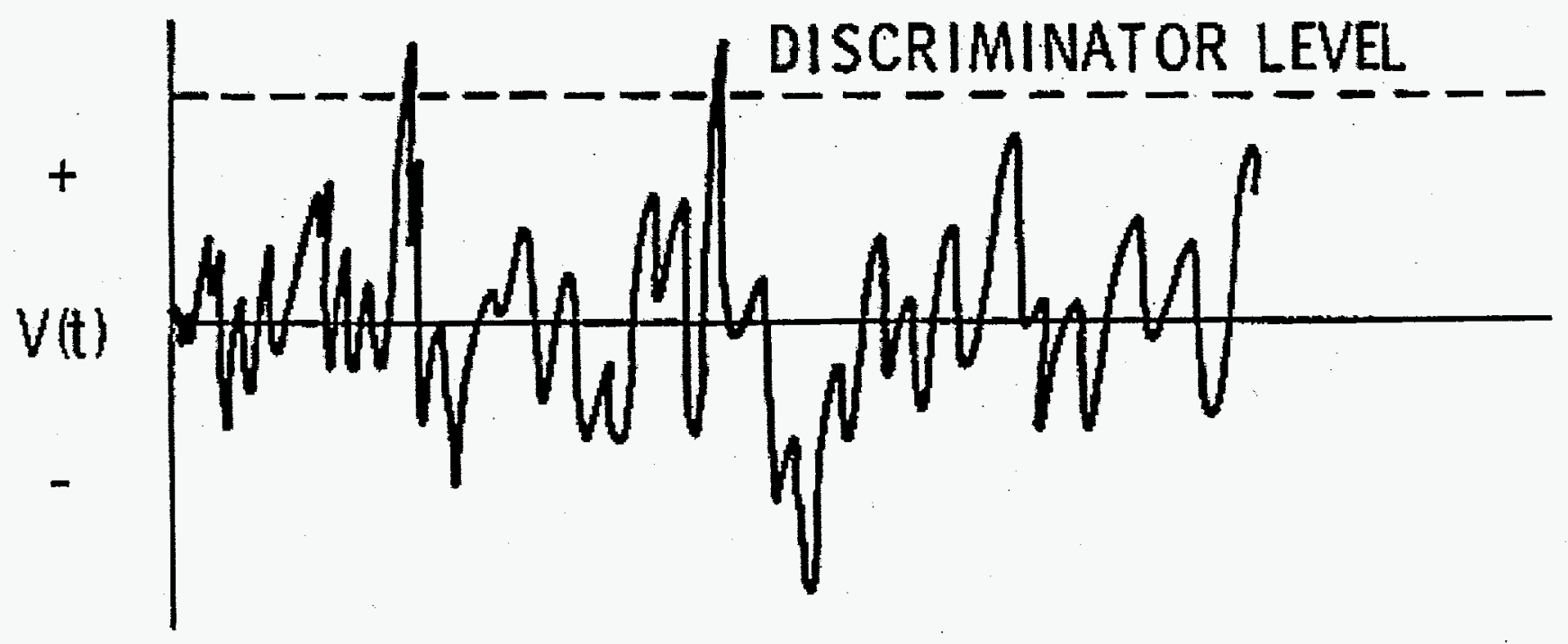

TIME

Figure 2.8. Typical Response of a ${ }^{3} \mathrm{He}$ Tube in a High Gamma-Ray Background. Note that the combined effect of many small gamma-ray pulses can occasionally pass the threshold for neutron pulse discrimination. 


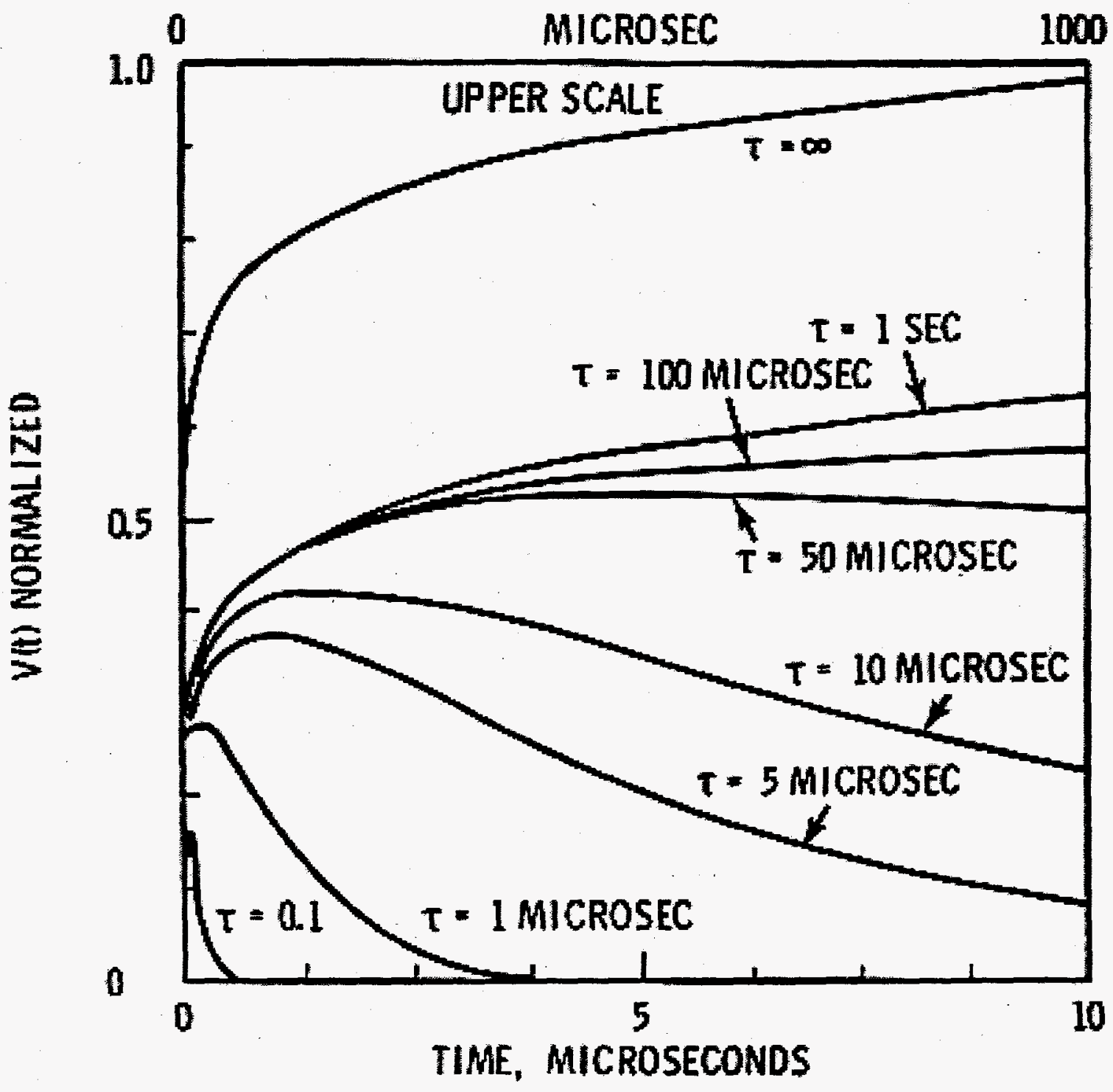

Figure 2.9 Neutron Pulse Shapes for a Variety of Pulse Shaping Times 
Time Interval Histogram $=252 \mathrm{Cf}$

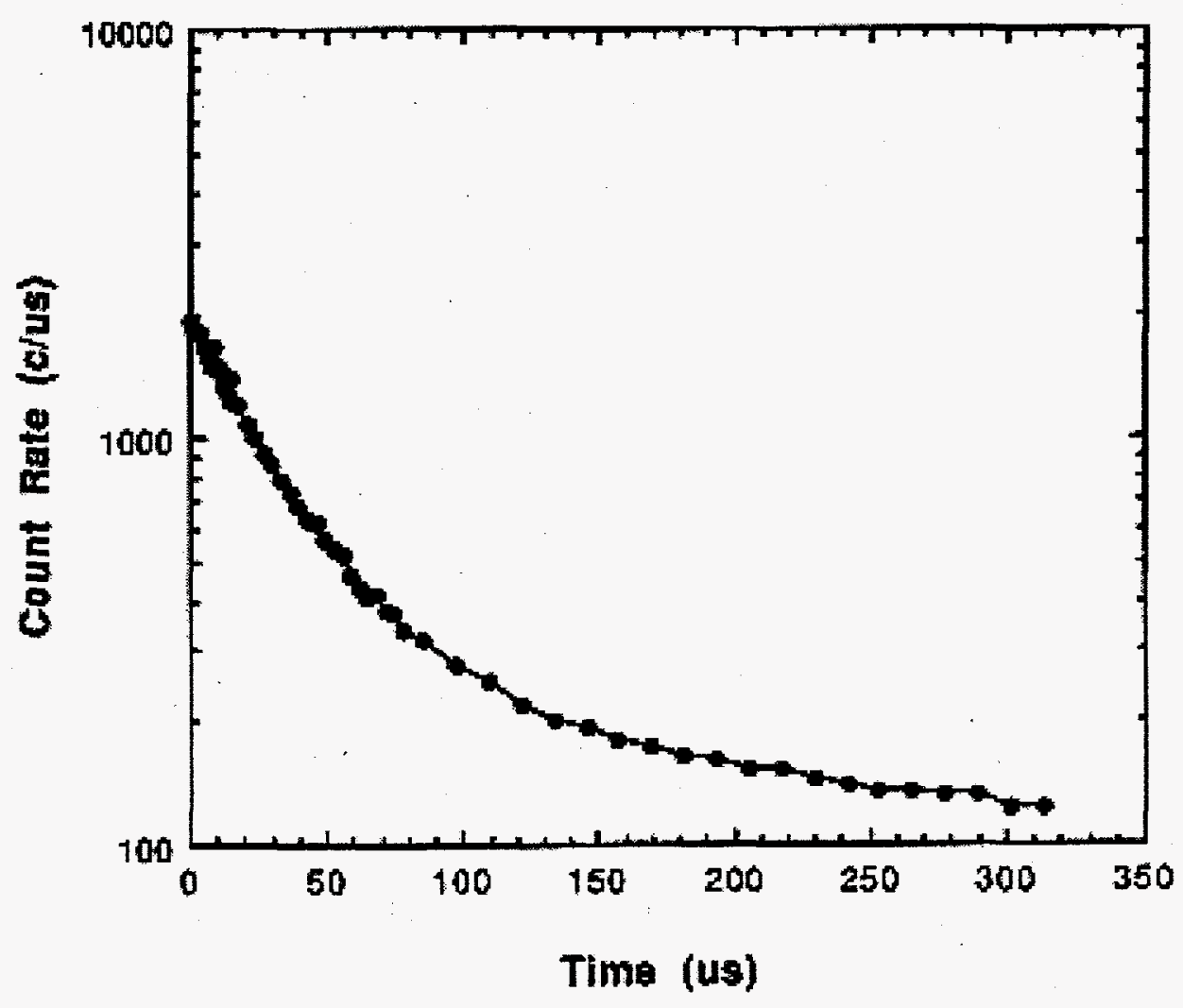

Figure 2.10 Histogram of All Time Intervals after Each Pulse in a ${ }^{252} \mathrm{Cf}$ Neutron Pulse Train 


\subsection{Capabilities}

\subsection{Theoretical Predictions}

The ability to confirm the presence of as little as 10 grams of weapons-grade plutonium requires a coincidence counter with an overall neutron detection efficiency of roughly $1 \%$. This conclusion follows from the following logic: Each gram of plutonium undergoes spontaneous fission at the rate of 28 fissions/second. For simplicitiy, we assume that 2 neutrons are emitted by each fission event.(this is a simplification, but a more accurate calculation yields similar results). The probability that a coincidence counter would successfully record any individual fission is $\epsilon^{2}$, where $\epsilon$ is the single neutron detection efficiency. In 1000 seconds (roughly 15 minutes), therefore, a counter with $\epsilon=0.01$ would observe 28 fissions from 10 grams of plutonium. The singles count rate expected from this system would be 6 counts per second. Assuming a coincidence time window of $50 \mu \mathrm{s}$, the accidental coincidence count rate can be neglected in this example. The natural background can also be neglected as it should lead to roughly 2 coincident counts in 15 minutes. Clearly, in this situation, a coincidence counter with $1 \%$ efficiency would achieve a sensitivity to quantities of plutonium as small as 10 grams in 1000 seconds. Sensitivity to $100 \mathrm{~g}$ or more is achieved in roughly 100 seconds.

A more careful analysis can be performed as follows. Suppose that a counter with efficiency $\epsilon$ is used to assay unknown materials with fission rate $F$ and single neutron emission rate $\alpha$. For this situation, the singles count rate will be approximately $S=\epsilon(2 F+\alpha)$, and the real coincident count rate will be roughly $R=\epsilon^{2} F$. Assuming that the coincident time window is $\tau$, the accidental coincidence rate will be $A=2 \tau S^{2}$. Assuming that a measurement of duration $T$ is performed, the overall fractional error $(E)$ in the measured real count rate will be

$$
E=(R T+2 A T)^{1 / 2} / R T
$$

The factor of 2 in the numerator of the previous equation accounts for the fact that the real count rate is obtained by subtracting $A$ from $R+A$. Using repeated substitution, the error can be rewritten as

$$
E=\left(\epsilon^{2} \mathrm{~F}+4 \tau \epsilon^{2}[2 \mathrm{~F}+\alpha]^{2}\right)^{1 / 2} / \epsilon^{2} \mathrm{FT} T^{1 / 2}
$$

which simplifies to

$$
E=\left(F+4 \tau[2 F+\alpha]^{2}\right)^{1 / 2} / \epsilon T^{1 / 2}
$$

For the example given in the previous paragraph, $E \approx 0.2$. Thus, the plutonium mass can only be estimated within $20 \%$ at the 1- $\sigma$ confidence level. Notice that for any measurement, the measurement error is inversely proportional to the detector efficiency. This rule of thumb provides a rapid way to compare the expected performance for the portable coincidence counter to the performance achieved with heavier, more efficient counters. 
A successful measurement is possible only when two separate conditions are met. First, a minimum number of real coincidence counts are necessary. This condition can effectively be written

$$
\epsilon^{2} \mathrm{FT} \gg 1
$$

A second condition is that the statistical uncertainty in the calculated real count rate must be smaller than the count rate itself. This condition can be written

$$
\mathrm{E} \ll 1.0
$$

Only when both conditions are satisfied is a successful measurement possible. It is wise to use these criteria to predict the necessary counter efficiency. It would be further possible to require a specific measurement accuracy such as $\mathrm{E}<0.05$ for a particular application.

\subsection{Experimental Tests}

\subsubsection{Effect of Multiple Wedges on Detection Efficiency}

The design implemented for testing the portable coincidence counter has ${ }^{3} \mathrm{He}$ tubes inside of wedgeshaped polyethylene segments. The cross sectional area of the moderating segments is $58 \mathrm{~cm}^{2}\left(9 \mathrm{in}^{2}\right.$.) Multiple detector "wedges" are used to make an array of detectors to increase detection efficiency. The wedge shape allows positioning of side-by-side detectors to form a straight line or to bend around a gradual curve. Velcro on the sides of the wedges allows them to attach to each other or to a strap around a barrel whose contents is being surveyed. Figure 3.1 shows a photograph of several wedges attached to a 30-gal barrel in a configuration similar to that used in many of these experiments.

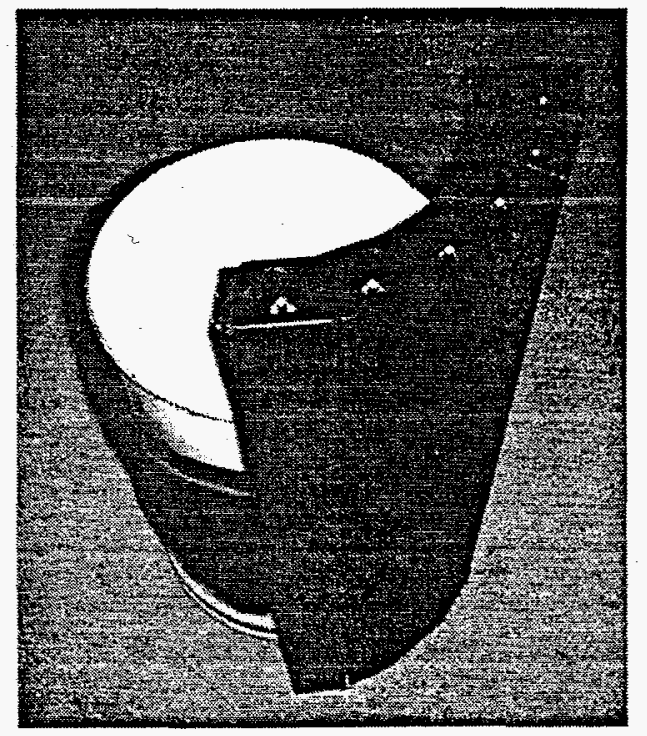

Figure 3.1 Detector Wedges Attached to Barrel 
The scattering of neutrons in the polyethylene of adjacent detector wedges enhances the detection efficiency more than that expected from merely increasing the detector volume. Table 3.1 shows the count rates obtained by placing a ${ }^{252} \mathrm{Cf}$ source at a distance of $61 \mathrm{~cm}$ ( 24 inches) away from varying numbers of detector segments. For these tests, the detectors are standing on their ends on a table to reduce the effects of neutron scatter from the floor, and the wedges are arranged to produce a straight row. As shown in the table, the count rate triples in going from one wedge to two wedges, rather than merely doubling as might be expected from the increased detector volume. This increase illustrates the benefit gained by placing wedges adjacent to each other rather than distributing them at isolated locations around a source.

Table 3.1 Effect of Multiple Detector Wedges on Count Rate

\begin{tabular}{|c|c|}
\hline (1) & (2) \\
\hline 1 & $0.91 \pm 0.02$ \\
\hline 2 & $2.88 \pm 0.02$ \\
\hline 3 & $5.25 \pm 0.03$ \\
\hline
\end{tabular}

\subsubsection{Detection Sensitivity for Neutrons}

For the neutron sensitivity tests, pulses from all detectors are summed before passing to a single, commercial preamplifier. Pulses passing a discrimination level of $2 \mathrm{~V}$ (with full-energy neutron pulses being $8 \mathrm{~V}$ ) are processed by conventional shift-register electronics manufactured by IRT Corporation (Figure 3.2). The commercial electronics and IRT shift register are used for collecting data because a sufficient number of new PNNL preamplifier/discriminators is not available, and our planned implementation of a GT 655 Time Interval Analyzer is not yet available.

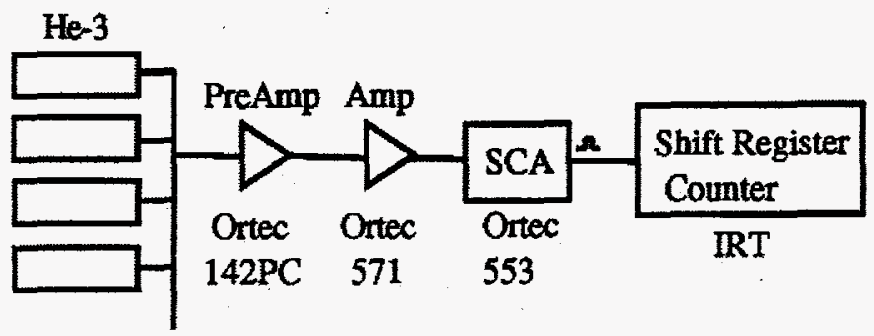

Figure 3.2 Electronics for Neutron Detection Efficiency Tests with Shift Register

Table 3.2 contains the data obtained using a ${ }^{252} \mathrm{Cf}$ source inside a barrel and various combinations of adjacent wedges outside the barrel. For most of the data, the total number of wedges is six, but for the final run shown, only four wedges are present. In the table, the notation " $3+3$ " indicates three adjacent wedges on one side of the barrel with three adjacent wedges on the opposite side of the barrel. Similarly, " $2+2+2$ " indicates two adjacent wedges placed at $120^{\circ}$ spacings around the barrel. The table shows the singles counts (single count in any detector), coincidence plus accidental counts in time intervals of $64 \mu \mathrm{s}$, and accidental 
counts (counts in $64-\mu \mathrm{s}$-wide window with 1-ms delay). Table 3.2 also shows the calculated neutron detection efficiencies based on a source emission rate of $20,600 \mathrm{n} / \mathrm{s}$. The largest neutron sensitivity is $8.8 \%$ for six adjacent wedges. Six wedges cover only about $30 \%$ of the area around a 30 -gal barrel, and other detectors could be added if necessary for greater detection efficiency.

Table 3.2 Neutron Counts Obtained from Detector Wedges Using Shift Register

\begin{tabular}{|c|c|c|c|c|c|c|}
\hline$y_{21}$ & 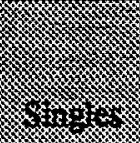 & 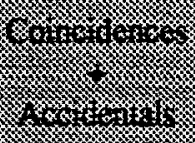 & (1) & $\left(y_{1}\right)$ & (1) & 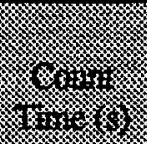 \\
\hline 6 & 543,215 & 29812 & 18901 & 10911 & 2.5 & 500 \\
\hline $3+3$ & 239,654 & 11573 & 7400 & 4173 & 2.2 & 500 \\
\hline $2+2+2$ & 213,607 & 8931 & 5790 & 3141 & 1.9 & 500 \\
\hline $1+1+1+1+1+1$ & 145,157 & 3965 & 2808 & 1157 & 1.25 & 500 \\
\hline 6 (background) & 30,187 & 58 & 42 & 16 & * & 1000 \\
\hline 4 & 161,776 & 5258 & 3406 & 1852 & 1.5 & 500 \\
\hline
\end{tabular}

\subsubsection{Gamma-Ray Sensitivity}

Although ${ }^{3} \mathrm{He}$ detectors primarily detect neutrons, high gamma-ray fluxes can produce apparent "neutron" counts as the result of gamma-ray pulse pile-up. The PNNL preamplifier/discriminator improves the gamma-ray rejection by performing rapid discrimination separately for each ${ }^{3} \mathrm{He}$ tube before outputs from multiple detectors are summed.

A single wedge containing a ${ }^{3} \mathrm{He}$ tube and preamplifier/discriminator provides the test data for gamma-ray sensitivity. The discriminator is set at $2 \mathrm{~V}$, and thermal neutron pulses have a full-energy magnitude of $8 \mathrm{~V}$. Equivalent data are also collected with commercial electronics in place of the PNNL preamplifier/discriminator for a comparison of the gamma-ray sensitivities. The gamma-ray source is ${ }^{137} \mathrm{Cs}$ in an pneumatically controlled system that is used to provide accurate exposure rates for health physics instrument calibration. The exposure rate is adjusted by changing the source-to-detector distance. Figure 3.3 shows the results obtained for various gamma-ray exposure rates for the two sets of electronics. Clearly the PNNL electronics provide lower gamma-ray count rates. The circuit used was a first engineering prototype; improvements should result in reduced gamma-ray response.

Comparing the neutron data in Table 3.2 with the gamma-ray data in Figure 3.3 gives an indication of the relative sensitivity of the detection system to the two types of radiation. The data in Table 3.2 are obtained at a location near a source storage vault, and the background for six wedges there is $30 \mathrm{c} / \mathrm{s}$. In comparison, the data in Figure 3.3 using the PNNL electronics show that the gamma-ray exposure rate required to produce a count rate of $30 \mathrm{c} / \mathrm{s}$ from six detectors would be about $0.7 \mathrm{R} / \mathrm{h}$. Thus, a very high gamma-ray background would be needed to interfere with any neutron measurement using this equipment. 


\subsubsection{Time Spectrum of Neutrons}

Figure 2.10 shows a time interval histogram measured using components of the prototype portable coincidence counter. a time analyzer (Stanford Research System 430) is used to collect the data, for which the "start" signal is derived from a single ${ }^{3} \mathrm{He}$ tube. The time analyzer sorts subsequent neutron pulses from the other wedges into consecutive time bins. Figure 2.10 shows the time spectrum obtained using a ${ }^{252} \mathrm{Cf}$ source and six detector wedges positioned around the source. Coincident neutrons emitted during spontaneous fission are characterized by increased counts at short time intervals after the start. This graph can be used to characterize the moderation time for the counter. Consequently, the optimal coincidence time window for use with a "shift register" coincidence analysis technique can be estimated.

Figure 3.4 shows, for comparison, the time spectrum obtained using a PuBe neutron source, which does not spontaneously fission and hence does not emit neutrons in coincidence. The flat time spectrum in the figure shows that the detected neutrons are uncorrelated in time, as expected from a nonfission source.

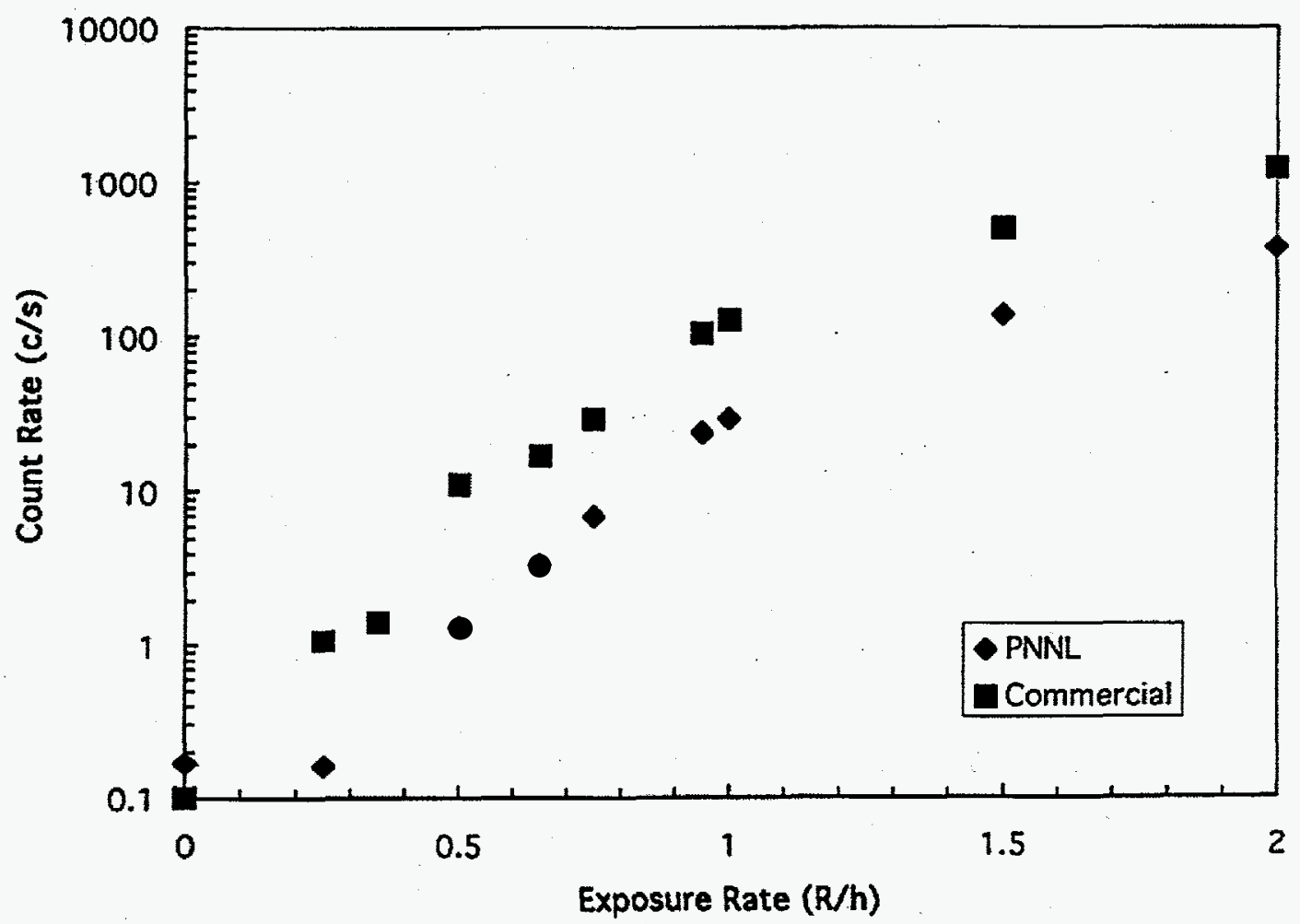

Figure 3.3 Gamma-Ray Count Rate of ${ }^{3} \mathrm{He}$ Detector for Exposure to Gamma Rays 


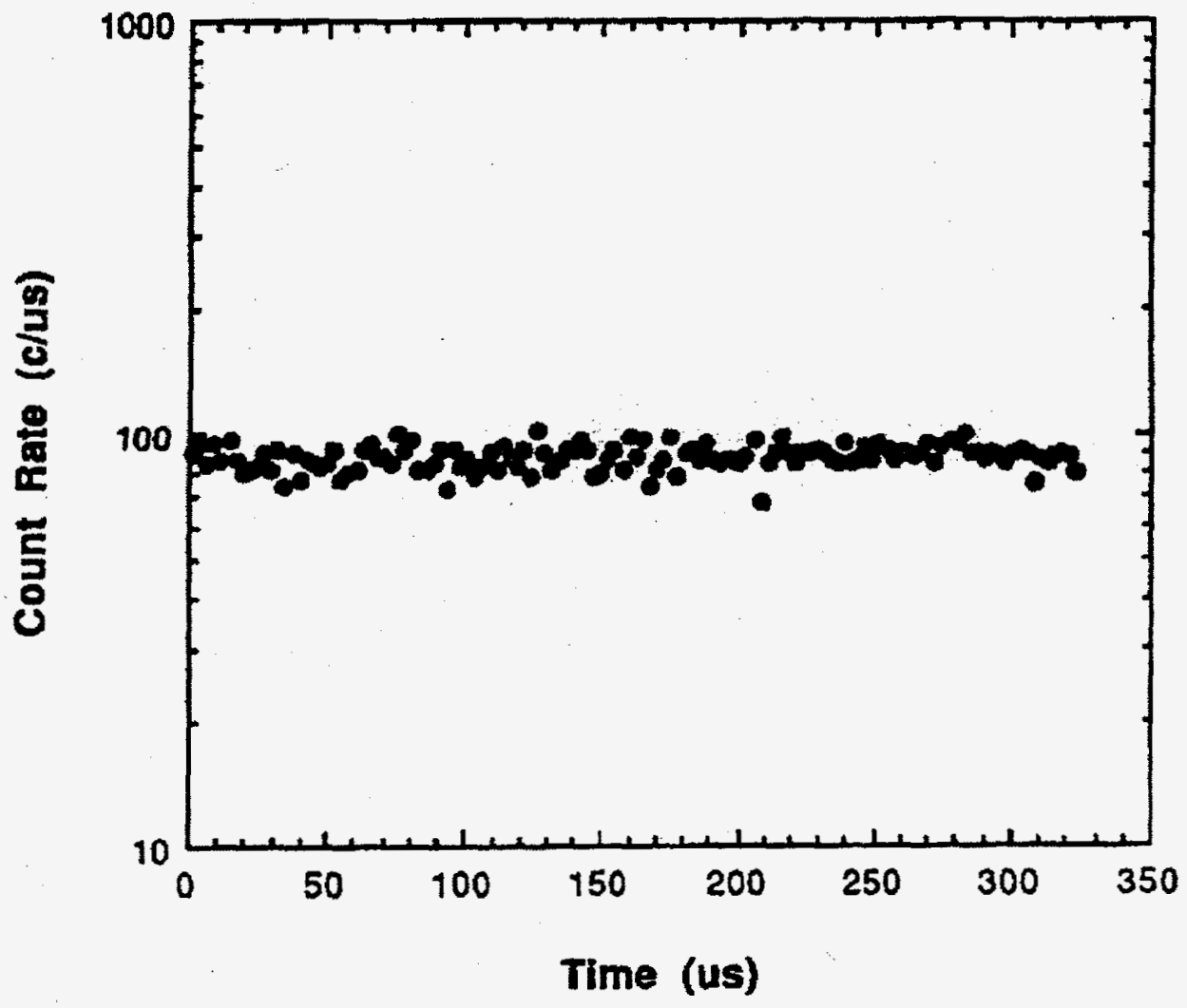

Figure 3.4 Time Spectrum from AmBe Showing Neutrons that Are Uncorrelated in Time 


\subsection{Discussion and Conclusion}

This report describes the design and construction of a portable coincidence counter that weighs less than $40 \mathrm{~kg}$ and is capable of confirming the presence of 10 grams of weapons-grade plutonium in a large container such as an ALR-8 (30-gal) drum). The design philosophy places a higher priority on portability, modularity, and flexibility than on detection efficiency. Thus, this counter should be ideally suited for remote, awkward, or unique measurements for which no existing counter is appropriate. The counter should be able to assay containers with a variety of shapes and sizes, and operate during gamma-ray exposures as high as $1 \mathrm{R} / \mathrm{h}$.

Although a number of applications exist for which optical fibers hold advantages over alternate technologies for neutron counting applications, ${ }^{3} \mathrm{He}$ detectors are the most suitable for the construction of a highly portable coincidence counter that must operate at relatively high gamma-ray exposures. Neutronsensitive fibers respond linearly to gamma-ray exposure according to the coefficient $1.5 \times 10^{-4}$ false neutron counts per $\mathrm{cm}$ of fiber per $\mathrm{mR} / \mathrm{h}$. As a result, unacceptably high gamma-ray-induced false neutron count rates will be experienced when a fiber-based counting system is subjected to high gamma-ray exposures. We conclude that ${ }^{3} \mathrm{He}$ detector technology is best suited for the construction of this specific coincidence counter that must operate in environments with a substantial gamma-ray exposure.

Numerical evidence is presented to support the conclusion that any such coincidence counter must be constructed from a fixed amount of moderator in the form of a number of relatively thick slabs. Distributing the moderator in the form of a thin "blanket" leads to poor detection efficiency and the need for a disadvantageously large coincident timing window. Further analysis describes how the counter's ultimate performance can be expected to depend on factors such as how efficiently it captures neutrons as well as the arrangement of the neutron detector and the moderator. A final design is described that allows flexible operation, yet satisfies weight limits applied to ensure portability. Fast electronics have been developed and miniaturized so the detector can operate in gamma-ray fields as strong as $1 \mathrm{R} / \mathrm{h}$.

Two theoretical criteria are derived that can be used to guide the use of this counting system. These criteria can be used to determine the counter efficiency and measurement time needed to ensure measurement success for any particular applications. Experiments confirm theoretical predictions concerning the detector's measurement capabilities.

\subsection{Acknowledgments}

We thank G.L. Carter, D.L. Haggard, and J.E. Tanner for helping with this research and providing radiation sources and equipment for testing the neutron coincidence counter. 


\subsection{References}

Armitage BH, PMJ Chard, S Croft, KP Lambert, and DJ Lloyd. 1994. "Characterization of the Harwell model N93 versatile passive neutron monitor," Nucl. Instru. Meth. Phys. Res. a, 353, p. 683.

Briesmeister JD (Ed). 1993. MCNP -- a general Monte Carlo n-particle transport code, version 4A, LA12625, Los Alamos National Laboratory, Los Alamos New Mexico.

Dytlewski N, MS Krick, and N Ennslin. 1993. "Measurement variances in thermal neutron coincidence counting," Nucl. Instru. Meth. Phys. Res. a, 327, p. 469.

Fehlau PE, HF Atwater, and KL Coop. 1990. "Neutron-chamber detectors and applications," Nucl. Instru. Meth. Phys. Res. a, 299, p. 29.

Halbig JK, SC Bourret, PR Collinsworth, WJ Hansen; and MS Krick. 1992. "Recent developments in multiplicity counting hardware at Los Alamos," IEEE Trans. Nucl. Sci., 39, p. 1326.

Stromswold DC, AJ Peurrung, PL Reeder, and RW Perkins. 1996. Measurement of ${ }^{235} U$ Content and Flow of UF ${ }_{6}$ Using Delayed Neutrons or Gamma-Rays Following Induced Fission, PNNL-11210, Pacific Northwest National Laboratory, Richland, Washington. 
Appendix A

Active Counting 


\section{Appendix A. Active Counting}

During active counting, a separate neutron source is placed near the container being assayed. The counting system must then measure the rate at which fission is induced by the external neutron source. In this way, materials such as uranium metal, which undergo induced fission but very little spontaneous fission, can be assayed. Designing a portable counter capable of active neutron counting is far harder than designing a passive counter. The relative difficulty of active and passive counter design can be seen in the nature of existing counting systems. The active systems are substantially heavier, more expensive, and more complicated than the corresponding passive systems.

The most important property of any active counter is that it must be able to effectively distinguish between the neutrons from the external source and the neutrons induced by fission within the container. Several methods are used to achieve this signal separation. First, a source of single neutrons exhibiting no coincident signal can be used for the external neutron source. Examples of such sources would be $(\alpha, n)$ sources such as americium-lithium (AmLi) or accelerator-based sources such as "d-t" neutron generators. Unfortunately, the very strong external source required would result in a large accidental coincidence rate that obscures the induced fission rate. Thus, additional means are needed to separate the external accidental signal and the induced fission signal. In many counters, energy discrimination is used; the $0.3-\mathrm{MeV}$ average energy of AmLi neutrons is substantially lower than the average energy of fission neutrons. Additionally, Pulsed neutron sources can be used to distinguish the induced fission rate (Rinard 1994). Pulsing can either be achieved by "shuffling" the external source back and forth between positions near and far from the container or by turning the neutron source off and on. The later method is easy to achieve using "d-t" neutron generators.

Many of the design principles commonly used for active counters are generally incompatible with lightweight, portable, and flexible counter design. Relatively large quantities of moderating material are required for active counter design. This moderator has, in essence, two separate functions. First, it is necessary to moderate (slow) the neutrons emitted by the external neutron source so that they are capable of effectively inducing fission. For this purpose, a thick moderator "cave" is often constructed. Secondly, as with passive counters, the moderator slows the fission neutrons from within the container so the counting system can detect neutrons effectively. Thus, the system's efficiency is significantly reduced if the moderator is removed to save weight. Additionally, the moderator acts as essential shielding for a shuffler when the external source is in the "far" position.

For the reasons outlined above, we conclude that conventional technology does not allow the construction of a lightweight, portable active neutron coincidence counter.

\section{Reference}

Rinard PM, KL Coop, NJ Nicholas, and HO Menlove. 1994. "Comparison of shuffler and differential dieaway technique instruments for the assay of fissile materials in 55-gallon waste drums," J. Nucl. Mater. Manage, 22, p. 28. 
Appendix B

The Detection Medium 


\section{Appendix B. The Detection Medium}

The two primary neutron detection technologies considered for constructing the portable coincidence counter are ${ }^{3} \mathrm{He}$ proportional counters ( ${ }^{3} \mathrm{He}$ tubes) and neutron-sensitive scintillating fiber optic glass. This section explains the analysis that led us to choose ${ }^{3} \mathrm{He}$ tubes as the more appropriate neutron detection technology for this application.

\section{B.1 Important Criteria}

A number of criteria need to be considered when choosing an optimal detection technology. The criteria that we consider are speed, cost, efficiency, physical form, and gamma-ray response. The discussion of gamma-ray response is reserved for a separate subsection because this characteristic proved to be the deciding factor in our decision to use ${ }^{3} \mathrm{He}$ tubes for neutron detection in this application.

\section{B.1.1 Speed}

Gas proportional counters, such as ${ }^{3} \mathrm{He}$ tubes, require roughly $1 \mu \mathrm{s}$ to collect ions following a neutron interaction. Neutron-sensitive scintillating optical fibers are able to process and record neutrondetection events in roughly $100 \mathrm{~ns}$. This higher speed gives a considerable advantage to fiber technology for certain neutron-detection applications. Examples of such applications include medical dosimetry, reactor flux monitoring, or any other high-rate application. Even in coincidence counting for nondestructive assay, highmultiplication fission events could lead to the detection of neutrons at the rate of 1 neutron per microsecond for a brief period of time. However, the type of lightweight, portable, low-efficiency counter assumed in this report will record neutron interactions at a rate $1 / 30$ as large as that in high-efficiency counters. As a result, the higher rate capability of neutron-sensitive fibers is not an important consideration for this application.

\section{B.1.2 Cost}

Analyzing the cost of any neutron detection system is notoriously complicated. Generally, the cost of any small system consists primarily of labor. As the size of any system grows, economies of scale should lead to a cost advantage for fiber-based neutron detection technologies. This occurs because of the time and labor required to start producing glass fiber in the PNNL fiber-draw facility. We conclude, however, that the cost of the detector itself is not an important consideration in the choice of technologies for a lightweight, low-efficiency counter.

\section{B.1.3 Efficiency}

The neutron detection efficiencies for neutron-sensitive scintillating fibers and ${ }^{3} \mathrm{He}$ gas-proportional counters are roughly comparable. (This statement refers to the detection medium itself, and not necessarily to systems built with these media. The following subsection, "form," explains this point.) A side-by-side row of 1-inch, 4-atmosphere ${ }^{3} \mathrm{He}$ tubes has the same thermal neutron capture cross section as 10 to 20 fiber layers, depending on the glass composition used. (This quantity of fiber is only 1.5 to $3.0 \mathrm{~mm}$ thick.) In most applications, a counting system's neutron detection efficiency depends more strongly on factors such as moderator weight, moderator arrangement, and source energy spectrum than on the simple neutron capture power of the detection medium. Efficiency, too, is not an important consideration in the technology choice 
for a coincidence counter that is constructed using relatively thick slabs of moderator. (The motivation for this design is explained in the next Section.)

\section{B.1.4 Form}

The physical form of the neutron-sensitive fiber-optic technology is well suited for a number of neutron counting applications where flexibility or compact size is important. The fibers are a solid, flexible, vibration-immune detection medium, whereas gas proportional counters are large, rigid, and may be vulnerable to electro-acoustical pickup. Although a collection of fibers has roughly the same inherent neutron capture "power" as a row of ${ }^{3} \mathrm{He}$ tubes, its physical form might provide a greater overall utility for several types of applications, such as the following:

- Environmental moderation: Often it is not necessary to construct a neutron counting system with its own moderator. It may be possible to rely upon natural moderators such as existing concrete, dirt, water, trees, or even people. In this case, the system's efficiency will depend primarily on the total area of the neutron detection medium. Fiber mats are easily configured to cover a large area of possibly irregular shapes.

- Thermal-neutron-specific detection: The localization of a neutron-emitting source from a long distance is frequently best accomplished using thermal-neutron-specific detection. A moderator-free detector will only measure thermal neutrons, from which a counter can be easily shielded in all directions except that of interest. Again, a large-area detection medium is critical for this purpose.

- Low-profile neutron detection: Any number of neutron-detection applications require a low-profile, lightweight neutron detection system. Fiber "ribbons" with compact electronics are again well suited for such applications.

A neutron coincidence counter intended for use in weapons dismantlement applications will necessarily contain a moderator in the form of relatively thick, discrete slabs. (Again, this conclusion is explained in Section III). Consequently, differences between the physical form of the different neutron detection technologies are of only secondary importance. The weight and size of any system will be determined by the moderator, rather than the detection medium.

\section{B.2 Gamma-Ray Tolerance}

For the application considered in this report, gamma-ray response is a critical factor to consider when comparing possible neutron-detection technologies. The physical mechanisms by which fibers and gas proportional counters respond to gamma-rays are completely distinct. A false neutron count is recorded in any gas proportional neutron counter primarily through the process of pulse pile-up. An important consequence of this is that the response of ${ }^{3} \mathrm{He}$ tubes to gamma-ray exposure depends in a highly nonlinear way on the intensity of the exposure. Previous studies have established that ${ }^{3} \mathrm{He}$ tubes can be made almost completely insensitive to gamma rays until gamma-ray exposure rates between $1 \mathrm{R} / \mathrm{h}$ and $10 \mathrm{R} / \mathrm{h}$ are reached (Crane and Baker 1991). The nature of a neutron proportional counter's response to gamma rays is illustrated by Figure B.1, which shows the measured response of a set ${ }^{10}{ }^{10} \mathrm{BF}_{3}$ tubes to a variable exposure from ${ }^{60} \mathrm{Co}$ gamma rays. This figure clearly shows that the false neutron count rate arising from gamma-ray exposure depends roughly exponentially on the exposure rate. This dependence is a fundamental characteristic of systems where pulse pile-up is the response mechanism. For the particular tubes used to 
collect the data shown in Figure B.1, virtually no response occurs until roughly $4 \mathrm{R} / \mathrm{h}$ of exposure is reached. These data, therefore, support our conclusion that a properly designed ${ }^{3} \mathrm{He}$-tube-based neutron coincidence counter will have a negligible response to gamma-ray exposures of less than roughly $1 \mathrm{R} / \mathrm{h}$. (A proper design requires, for example, separate threshold discriminators for each tube and rapid pulse-shaping circuitry.)

The response of neutron-sensitive fibers to gamma-ray exposure is fundamentally different from that of ${ }^{3} \mathrm{He}$ tubes. The response is linear (except for exceptionally intense exposures) because pile-up of two or more separate gamma rays is not required to record a false neutron count. Occasionally, a particular gamma ray will mimic a neutron interaction through a combination of the three following processes:

1. The gamma-ray must interact within or near the fibers themselves, producing a fast electron probably through either the Compton or photoelectric processes.

2. This fast electron must travel a path that allows it to travel a relatively large distance within the scintillating core of one or more fibers. This might happen if the electron were traveling down the length of a fiber, or perhaps if the electron traveled a substantial distance through a "bundle" of active fibers. Cherenkov radiation within the glass of a PMT may also be a mechanism for gamma ray-interaction.

3. Photons must be generated in sufficient number for at least one to be recorded at each end of the fiber bundle and for a threshold number to be recorded in total.

Each of the processes described above is statistically unlikely. This is why neutron-sensitive fibers manage to achieve a highly successful discrimination between neutron events and gamma-ray events when total gamma-ray exposures are modest. However, the important point here is that the response of scintillating fibers to any gamma-ray exposure will be linearly proportional to the intensity of the exposure. We also expect that the response of any fiber system to gamma rays will be proportional to the amount of fiber used, which is conveniently measured as the linear length of fiber present. This linear gamma-ray response can actually be an advantage for many applications where simultaneous neutron and gamma-ray detection is desired. Fiber-based neutron counting systems often count gamma-ray events separately by counting those events that fail to meet the neutron threshold, but which still meet the requirement for coincidence at both ends of a particular fiber.

The response of fibers to gamma-ray exposure is most easily characterized in terms of a coefficient reflecting the physics discussed above. We define the coefficient $\Gamma$ as follows:

$$
\Gamma=(\text { false count rate }) /(\gamma \text {-ray exposure })(\text { total fiber length) }
$$

where a "false count" is any neutron event recorded by the counter that arises from gamma-ray interactions. This coefficient has been experimentally measured for five different fiber systems designed for weapons detection at a distance (two systems), medical dosimetry, reactor flux monitoring, and safeguards. The results are tabulated in Table B.1. As expected, all fiber systems have a gamma-ray sensitivity that can be characterized by a single value for the coefficient $\Gamma$. The most appropriate value indicated by Table B.1 is roughly $1.5 \times 10^{-4}$ false neutron counts per second per $\mathrm{mR} / \mathrm{h}$ per $\mathrm{cm}$ of fiber. 

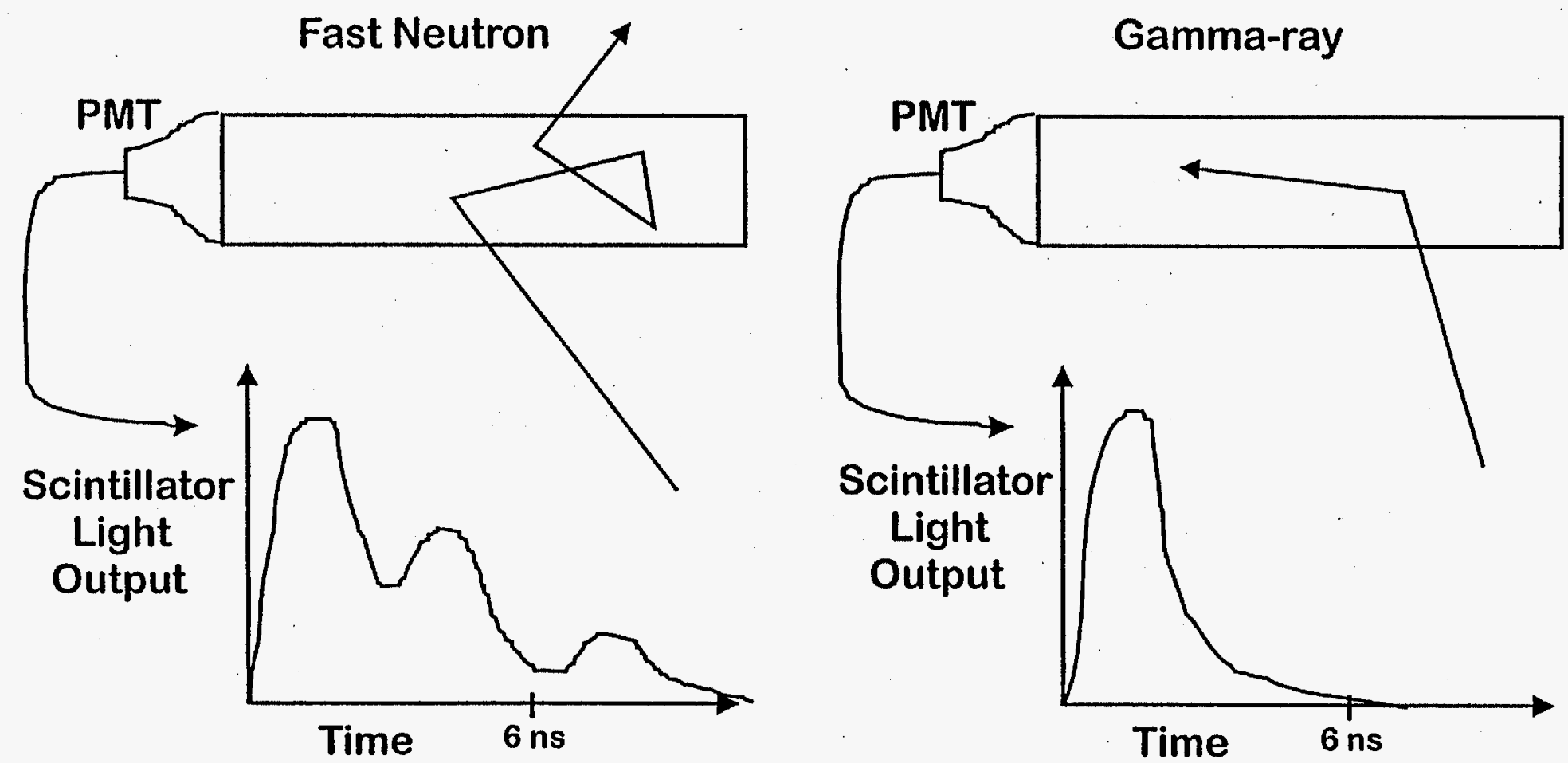

Figure B.1 False Neutron Count Rate in a Set of ${ }^{10} \mathrm{BF}_{3}$ Tubes as a Function of Gamma-Ray Exposure Rate. Below $4 \mathrm{R} / \mathrm{h}$, the count rate is indistinguishable from background. 
Table B.1 Gamma-Ray Response Data for Five Different Fiber-Based Neutron Counters. A threshold of four photoelectrons (with at least one at each end of the fiber bundle) was used for all of these data.

\begin{tabular}{|c|c|c|c|c|}
\hline (1) & 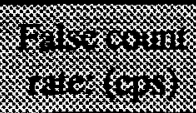 & (2) & 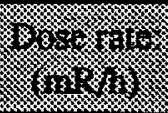 & $(x)$ \\
\hline Reactor monitor & 5.3 & $3.5 \times 10^{5}$ & 0.04 & $3.8 \times 10^{-4}$ \\
\hline Roadway monitor & 12 & $2 \times 10^{6}$ & 0.043 & $1.4 \times 10^{-4}$ \\
\hline $\begin{array}{l}\text { Human presence } \\
\text { detector }\end{array}$ & 2.2 & $3.1 \times 10^{5}$ & 0.048 & $1.5 \times 10^{-4}$ \\
\hline Dosimeter & 30,000 & 6 & $5.7 \times 10^{7}$ & $9 \times 10^{-5}$ \\
\hline $\begin{array}{l}\text { Large area neutron } \\
\text { detector }\end{array}$ & 2.5 & $1.1 \times 10^{6}$ & 0.033 & $7 \times 10^{-5}$ \\
\hline
\end{tabular}

The gamma-ray response data for the first, second, and third neutron counting systems shown in Table B.1 were acquired at PNNL specifically for this report. Each of these tests used a ${ }^{60} \mathrm{Co}$ source that was placed at a sufficient distance from the fiber system that a uniform gamma-ray exposure could be assumed. The neutron response of the "human presence detector" and the "reactor monitor" were also studied in detail to verify optimal counting-system operation. The neutron-sensitive fiber dosimeter was tested at Washington State University in Pullman at a research reactor (Bliss et al. 1996). This test is particularly valuable because it involves far smaller amounts of fiber and higher gamma-ray exposures. The gamma-ray response of the "large area neutron detector" is calculated from previous data describing the comparative efficiency of this system for detecting neutrons and gamma-rays. It is necessary to assume a value for this system's absolute neutron detection efficiency to infer the data contained in Table B.1. An efficiency of 25\% was assumed. All of the data in Table B.1 were acquired with a threshold of 4 photoelectrons required for neutron events. The similarity in the gamma-ray response coefficients for five separate neutron detection systems implies that gamma-ray response is an inherent property of the fibers themselves, rather than a characteristic of each particular electronics configuration.

\section{B.3 Application to Portable Coincidence Counting}

The consequences of the fiber's gamma-ray response characteristics are serious for the application of coincidence counting in high gamma-ray backgrounds. A counter designed to be $1 \%$ efficient will necessarily contain a total of roughly $2 \times 10^{6} \mathrm{~cm}$ of neutron-sensing fiber. If we further assume that such a counter will need to operate in gamma-ray exposures as high as $100 \mathrm{mR} / \mathrm{h}$, we find that the false neutron count rate arising from gamma-ray interactions is roughly 30,000 counts per second. Numerical neutron transport calculations indicate that the neutron moderation die-away time for the counter is roughly $30 \mu \mathrm{s}$. To detect coincident pairs of neutrons arising from fission events efficiently, a coincidence time window of $50 \mu \mathrm{s}$ or more will be required. Clearly, this counting system will fail to measure real coincident neutron pairs among the background rate of 30,000 cps. Under such conditions, the counting system will be "saturated" with false coincident counts. (The average interval between false neutrons is $33 \mu \mathrm{s}$, which is less than the required coincidence window.) Even at a gamma-ray exposure rate of $10 \mathrm{mR} / \mathrm{h}$, the false neutron background rate is 
3000 cps. The expected "accidental" coincidence count rate would be roughly 1000 coincident counts per second. (The accidental coincidence rate is $2 \tau \mathrm{S}^{2}$, where $\tau$ is the coincidence time window and $S$ is the rate of neutron events.) The real signal of 2.8 coincident counts per second from $1 \mathrm{~kg}$ of weapons-grade plutonium will be completely lost in a background of accidental coincidences.

The fiber response to gamma-rays can be reduced by increasing the electronics detection threshold. Although a higher threshold will dramatically reduce the false neutron count rate, the accompanying reduction in neutron detection efficiency is highly undesirable. We conclude, therefore, that ${ }^{3} \mathrm{He}$ tube technology is the most suitable for constructing a highly portable coincidence counter that must operate in environments with a substantial gamma-ray exposure.

\section{References}

Bliss M, RA Craig, DS Sunberg, YC Harker, JR Hartwell, and JR Venhuizen. "Progress Towards Development of Real-Time Dosimetry for BNCT." To be published in: Proceedings for The Seventh International Symposium on Neutron Capture Therapy for Cancer, Zurich, September 4-7, 1996.

Crane TW and MP Baker. 1991. Passive Nondestructive Assay of Nuclear Materials, D Riley, N Ensslin, and H Smith, Jr. (Eds.), Chap 13, p. 384-386. 


\section{Appendix C}

\section{Optimization of Fiber-Detector Design}




\section{Appendix C. Optimization of Fiber-Detector Design}

A primary question that any detector designer faces is what quantity of neutron capture isotope to use in a detector. For ${ }^{3} \mathrm{He}$ tubes, a pressure and tube diameter must be selected. For ${ }^{6} \mathrm{Li}$-containing scintillating fibers, some number of fiber layers must be used. In both cases, cost and complexity increase as more of the capture isotope is used. Thus, we performed computations designed to reveal the effect of changing the quantity of capture isotope on the detector efficiency. Figure C.1 shows these results. We have confirmed that the results for ${ }^{3} \mathrm{He}$ are qualitatively very similar, provided that equal total cross-sections are used. (Each atom of ${ }^{3} \mathrm{He}$ has a cross section equivalent to approximately that of $5.7^{6} \mathrm{Li}$ atoms.) Notice that the efficiency depends roughly logarithmically on the quantity of neutron capture isotope. Thus, increasing detector efficiency by increasing the quantity of neutron capture isotope beyond a certain point is prohibitively expensive and impractical. Figure C.2 shows the ratio of the number of ${ }^{6} \mathrm{Li}$ captures to hydrogen captures as a function of ${ }^{6} \mathrm{Li}$ content. Note the power-law nature of this relationship.

Another major question for detector design concerned the relative advantage that could be gained by dispersing scintillating fibers within a moderating matrix. (A similar dispersal would be hard to accomplish using ${ }^{3} \mathrm{He}$ tubes.) To answer this question, we calculated the efficiency of a cylindrically shaped detector surrounding a fission source as a function of the fiber arrangement. The total quantity of ${ }^{6} \mathrm{Li}$ was fixed; i.e., if four fiber layers were used instead of two, then each layer would contain half as much ${ }^{6} \mathrm{Li}$. The moderator in each case was $5 \mathrm{~cm}$ thick. Figure C.3 shows the results of these calculations. For each case, the assumed total quantity of ${ }^{6} \mathrm{Li}$ was divided into some number of discrete fiber layers, which were assumed to be equally spaced within the moderator. Only in some cases, a fiber layer was placed at the inside of the moderating cylinder. A fiber layer was never placed at the outside. (Neutrons leaving the moderating cylinder through the inside must re-enter the detector, but neutrons leaving through the outside cannot.) The horizontal lines in Figure C.3 denote the ${ }^{6} \mathrm{Li}$ and hydrogen capture probabilities for the case where the ${ }^{6} \mathrm{Li}$ is uniformly dispersed. (This is equivalent to an infinite number of fiber layers.) Note that a detector with a single fiber layer is at least $75 \%$ as efficient as a detector with uniformly dispersed ${ }^{6} \mathrm{Li}$. Thus, the ability to disperse fibers constitutes an advantage, but not a tremendous one. The importance of fiber dispersal will be more significant for thicker moderating slabs. 


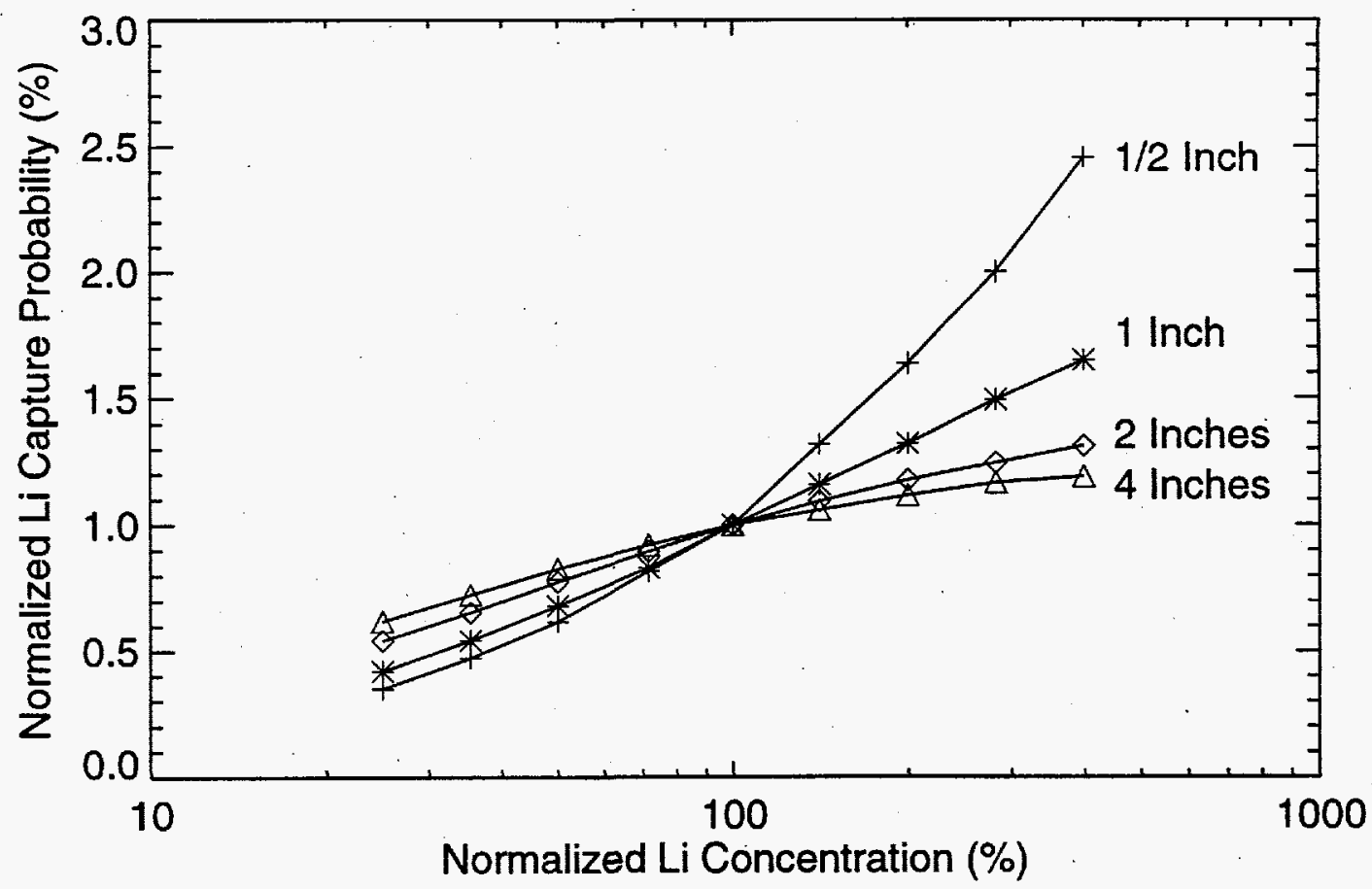

Figure C.1 Normalized Fiber Detector Efficiency as a Function of the Detector's Normalized 'Li Content for Four Different Thicknesses. The "normal" " $\mathrm{Li}$ content corresponds to three bilayers of fiber (with a standard glass composition) in a detector that was $6.25 \mathrm{~cm}$ thick. Notice that the effect of changing the ${ }^{6} \mathrm{Li}$ content is least for thick detectors.

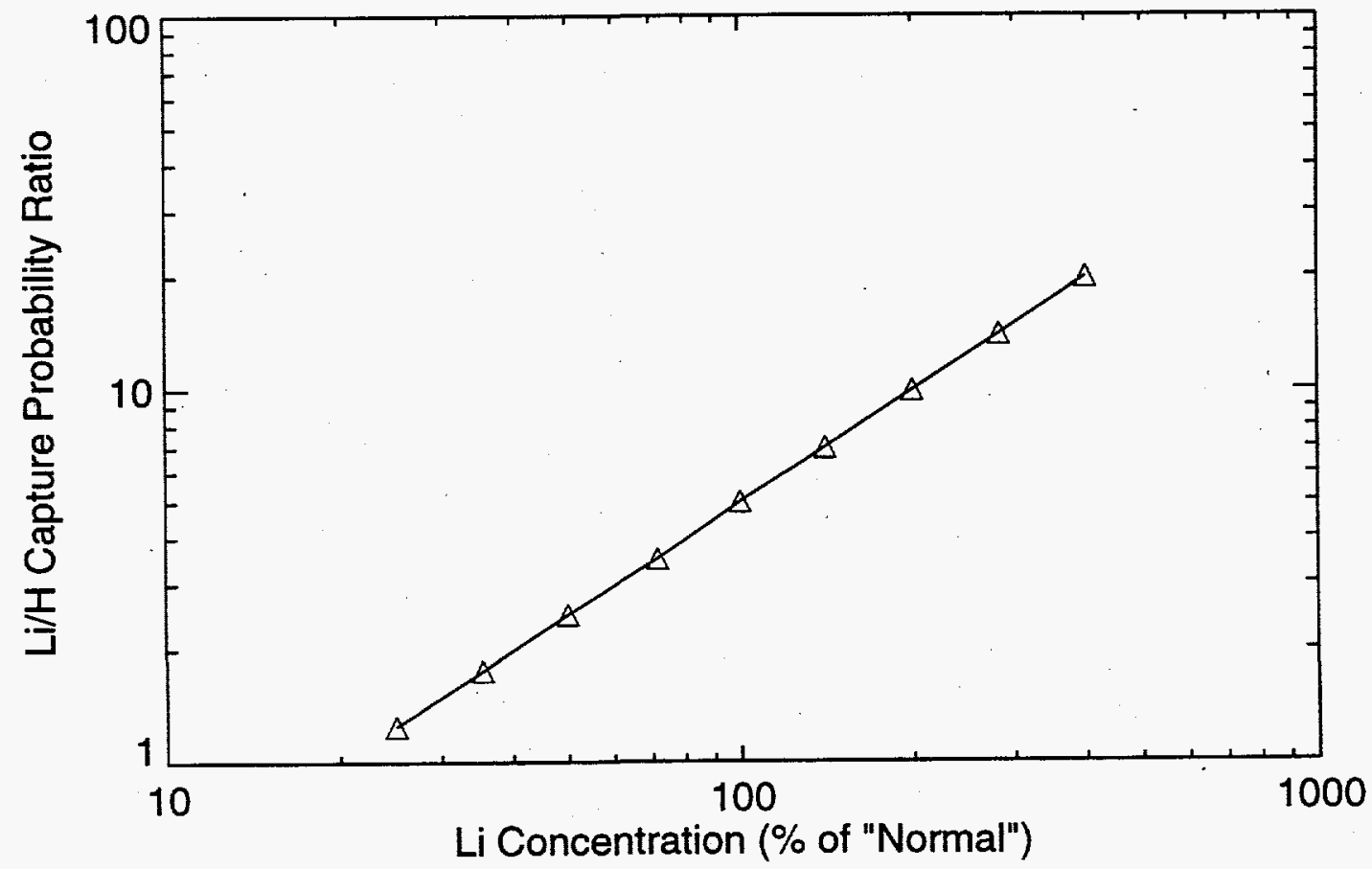

Figure C.2 Ratio of ${ }^{6} \mathrm{Li}$ Capture Probability to Hydrogen Capture Probability as a Function of the Normalized ${ }^{6} \mathrm{Li}$ Detector Content for a $2.5-\mathrm{cm}$-Thick Detector. Note that both axes are logarithmic. 


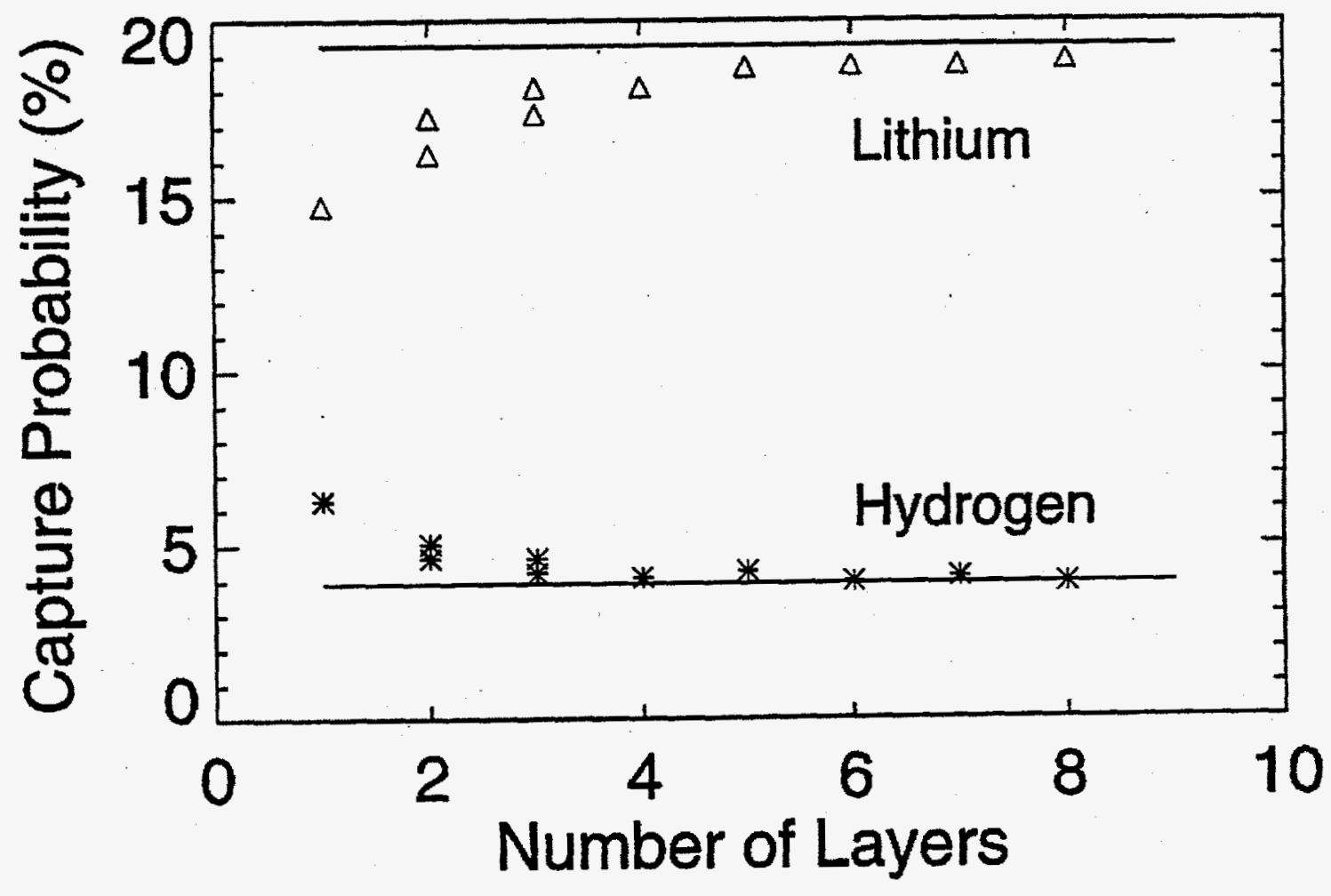

Figure C.3 ${ }^{6} \mathrm{Li}$ and Hydrogen Capture Probability as a Function of Optical Fiber Arrangement. The efficiency of a detector is essentially the same as the probability for ${ }^{6} \mathrm{Li}$ capture. The total quantity of ${ }^{6} \mathrm{Li}$ was fixed, but subdivided into a number of equally spaced segments or layers. As usual, neutrons with a uranium fission energy spectrum were assumed. 


\section{Distribution}

No. of

Copies

Offsite

2 DOE Office of Scientific and Technical Information

$7 \quad D O E / H Q$

M.F. O'Connell NN-20

D.P. Spears $\quad$ NN-20

M.J. Newman $\quad \mathrm{NN}-42$

A. J. Bieniawski NN-42

R.C. Cherry NN-44

A.B. Whitworth NN-44

D. Crawford NN-50

Forrestal Bldg.

U.S. Department of Energy

1000 Independence Ave., SW

Washington DC 20585

D.W. Crawford NN-512

U.S. Department of Energy

19901 Germantown Road

Germantown MD 20874-1290

PANTEX

K. Franklin

Applied Technology Division

Pantex Plant

Mason \& Hanger Corporation

P.O. Box 30020

Amarillo TX 79120-0020

R.C. McGilvary III

Safeguards, Security \& Fire Protection

Division

Pantex Plant

Mason \& Hanger Corporation

P.O. Box 30020

Amarillo TX 79120-0020
No, of

Copies

Onsite

47 Pacific Northwest National Laboratory

O.P. Amacker

M. Bliss

K.R. Byers

W.C. Cliff

R.A. Craig

S.M. Bowyer (5)

G.B. Dudder (5)

D.L. Haggard

M.A. Knopf

B.J. Merrill

M.E. Panisko

R.W. Perkins

A.J. Peurrung (5)

P.L. Reeder

D.E. Robertson

R.J. Sorenson

D.C. Stromswold (5)

D.S. Sunberg

J.E. Tanner

H. Undem (5)

R.A. Warner

N.A. Wogman

Technical Report Files (5)

Distr. 1 